\title{
FLATNESS AND DEFECT OF NONLINEAR SYSTEMS: INTRODUCTORY THEORY AND EXAMPLES *
}

\author{
Michel Fliess $^{\dagger} \quad$ Jean Lévine $^{\ddagger} \quad$ Philippe Martin $^{\S} \quad$ Pierre Rouchon $^{\mathbb{I}}$ \\ CAS internal report A-284, January 1994.
}

We introduce flat systems, which are equivalent to linear ones via a special type of feedback called endogenous. Their physical properties are subsumed by a linearizing output and they might be regarded as providing another nonlinear extension of Kalman's controllability. The distance to flatness is measured by a non-negative integer, the defect. We utilize differential algebra which suits well to the fact that, in accordance with Willems' standpoint, flatness and defect are best defined without distinguishing between input, state, output and other variables. Many realistic classes of examples are flat. We treat two popular ones: the crane and the car with $n$ trailers, the motion planning of which is obtained via elementary properties of planar curves. The three non-flat examples, the simple, double and variable length pendulums, are borrowed from nonlinear physics. A high frequency control strategy is proposed such that the averaged systems become flat.

*This work was partially supported by the G.R. "Automatique" of the CNRS and by the D.R.E.D. of the "Ministère de l'Éducation Nationale". 


\section{Introduction}

We present here five case-studies: the control of a crane, of the simple, double and variable length pendulums and the motion planning of the car with $n$-trailers. They are all treated within the framework of dynamic feedback linearization which, contrary to the static one, has only been investigated by few authors (Charlet et al. 1989, Charlet et al. 1991, Shadwick 1990). Our point of view will be probably best explained by the following calculations where all vector fields and functions are real-analytic.

Consider

$$
\dot{x}=f(x, u) \quad\left(x \in \mathbb{R}^{n}, \quad u \in \mathbb{R}^{m}\right),
$$

where $f(0,0)=0$ and $\operatorname{rank} \frac{\partial f}{\partial u}(0,0)=m$. The dynamic feedback linearizability of (1) means, according to (Charlet et al. 1989), the existence of

1. a regular dynamic compensator

$$
\left\{\begin{array}{l}
\dot{z}=a(x, z, v) \\
u=b(x, z, v)
\end{array} \quad\left(z \in \mathbb{R}^{q}, \quad v \in \mathbb{R}^{m}\right)\right.
$$

where $a(0,0,0)=0, b(0,0,0)=0$. The regularity assumption implies the invertibility ${ }^{1}$ of system (2) with input $v$ and output $u$.

2. a diffeomorphism

$$
\xi=\Xi(x, z) \quad\left(\xi \in \mathbb{R}^{n+q}\right)
$$

such that (1) and (2), whose $(n+q)$-dimensional dynamics is given by

$$
\left\{\begin{aligned}
\dot{x} & =f(x, b(x, z, v)) \\
\dot{z} & =a(x, z, v)
\end{aligned}\right.
$$

becomes, according to (3), a constant linear controllable system $\dot{\xi}=F \xi+G v$.

Up to a static state feedback and a linear invertible change of coordinates, this linear system may be written in Brunovsky canonical form (see, e.g., (Kailath 1980)),

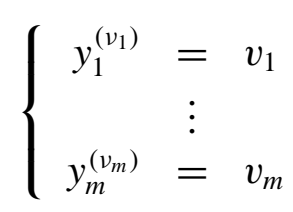

where $v_{1}, \ldots, v_{m}$ are the controllability indices and $\left(y_{1}, \ldots, y_{1}^{\left(\nu_{1}-1\right)}, \ldots, y_{m}, \ldots, y_{m}^{\left(v_{m}-1\right)}\right)$ is another basis of the vector space spanned by the components of $\xi$. Set $Y=\left(y_{1}, \ldots, y_{1}^{\left(\nu_{1}-1\right)}, \ldots, y_{m}, \ldots, y_{m}^{\left(v_{m}-1\right)}\right)$;

\footnotetext{
${ }^{1}$ See (Li and Feng 1987) for a definition of this concept via the structure algorithm. See (Di Benedetto et al. 1989, Delaleau and Fliess 1992) for a connection with the differential algebraic approach.
} 
thus $Y=T \xi$ where $T$ is an invertible $(n+q) \times(n+q)$ matrix. Otherwise stated, $Y=T \Xi(x, z)$. The invertibility of $\Xi$ yields

$$
\left(\begin{array}{c}
x \\
z
\end{array}\right)=\Xi^{-1}\left(T^{-1} Y\right)
$$

Thus from (2) $u=b\left(\Xi^{-1}\left(T^{-1} Y\right), v\right)$. From $v_{i}=y_{i}^{\left(v_{i}\right)}, i=1, \ldots, m, u$ and $x$ can be expressed as real-analytic functions of the components of $y=\left(y_{1}, \ldots, y_{m}\right)$ and of a finite number of their derivatives:

$$
\left\{\begin{array}{l}
x=\mathcal{A}\left(y, \dot{y}, \ldots, y^{(\alpha)}\right) \\
u=\mathcal{B}\left(y, \dot{y}, \ldots, y^{(\beta)}\right)
\end{array} .\right.
$$

The dynamic feedback (2) is said to be endogenous if, and only if, the converse holds, i.e., if, and only if, any component of $y$ can be expressed as a real-analytic function of $x, u$ and a finite number of its derivatives:

$$
y=\mathcal{C}\left(x, u, \dot{u}, \ldots, u^{(\gamma)}\right) .
$$

Note that, according to (4), this amounts to expressing $z$ as a function of $\left(x, u, \dot{u}, \ldots, u^{(\rho)}\right)$ for some $\rho$. In other words, the dynamic extension does not contain exogenous variables, which are independent of the original system variables and their derivatives. This justifies the word endogenous. Note that quasi-static feedbacks, introduced in the context of dynamic input-output decoupling (Delaleau and Fliess 1992), share the same property.

A dynamics (1) which is linearizable via such an endogenous feedback is said to be (differentially) flat; $y$, which might be regarded as a fictitious output, is called a linearizing or flat output. The terminology flat is due to the fact that $y$ plays a somehow analogous role to the flat coordinates in the differential geometric approach to the Frobenius theorem (see, e.g., (Isidori 1989, Nijmeijer and van der Schaft 1990)). A considerable amount of realistic models are indeed flat. We treat here two case-studies, namely the crane (D’Andréa-Novel and Lévine 1990, Marttinen et al. 1990) and the car with $n$ trailers (Murray and Sastry 1993, Rouchon et al. 1993a). Notice that the use of a linearizing output was already known in the context of static state feedback (see (Claude 1986) and (Isidori 1989, page 156)).

One major property of differential flatness is that, due to formulas (5) and (6), the state and input variables can be directly expressed, without integrating any differential equation, in terms of the flat output and a finite number of its derivatives. This general idea can be traced back to works by D. Hilbert (Hilbert 1912) and E. Cartan (Cartan 1915) on under-determined systems of differential equations, where the number of equations is strictly less than the number of unknowns. Let us emphasize on the fact that this property may be extremely usefull when dealing with trajectories: from $y$ trajectories, $x$ and $u$ trajectories are immediately deduced. We shall detail in the sequel various applications of this property from motion planning to stabilization of reference trajectories. The originality of our approach partly relies on the fact that the same formalism applies to study systems around equilibrium points as well as around arbitrary trajectories.

As demonstrated by the crane, flatness is best defined by not distinguishing between input, state, output and other variables. The equations moreover might be implicit. This standpoint, which matches well with Willems' approach (Willems 1991), is here taken into account by utilizing differential 
algebra which has already helped clarifying several questions in control theory (see, e.g., (Diop 1991, Diop 1992, Fliess 1989, Fliess 1990a, Fliess and Glad 1993)).

Flatness might be seen as another nonlinear extension of Kalman's controllability. Such an assertion is surprising when having in mind the vast literature on this subject (see (Isidori 1989, Nijmeijer and van der Schaft 1990) and the references therein). Remember, however, Willems' trajectory characterization (Willems 1991) of linear controllability which can be interpreted as the freeness of the module associated to a linear system (Fliess 1992). A linearizing output now is the nonlinear analogue of a basis of this free module.

We know from (Charlet et al. 1989) that any single-input dynamics which is linearizable by a dynamic feedback is also linearizable by a static one. This implies the existence of non-flat systems which verify the strong accessibility property (Sussmann and Jurdjevic 1972). We introduce a nonnegative integer, the defect, which measures the distance from flatness.

These new concepts and mathematical tools are providing the common formalism and the underlying structure of five physically motivated case studies. The first two ones, i.e., the control of a crane and the motion planning of a car with $n$-trailers, which are quite concrete, resort from flat systems. The three others, i.e., the simple and double Kapitsa pendulums and the variable-length pendulum exhibit a non zero defect.

The characterization of the linearizing output in the crane is obvious when utilizing a non-classic representation, i.e., a mixture of differential and non-differential equations, where there are no distinction between the system variables. It permits a straightforward tracking of a reference trajectory via an open-loop control. We do not only take advantage of the equivalence to a linear system but also of the decentralized structure created by assuming that the engines are powerful with respect to the masses of the trolley and the load.

The motion planning of the car with $n$-trailer is perhaps the most popular example of path planning of nonholonomic systems (Laumond 1991, Murray and Sastry 1993, Monaco and Normand-Cyrot 1992, Rouchon et al. 1993a, Tilbury et al. 1993, Martin and Rouchon 1993, Rouchon et al. 1993b). It is a flat system where the linearizing output is the middle of the axle of the last trailer. Once the linearizing output is determined, the path planning problem becomes particularly easy: the reference trajectory as well as the corresponding open-loop control can be expressed in terms of the linearizing output and a finite number of its derivatives. Let us stress that no differential equations need to be integrated to obtain the open-loop control. The relative motions of the various components of the system are then obtained thanks to elementary geometric properties of plane curves. The resulting calculations, which are presented in the two-trailer case, are very fast and have been implemented on a standard personal microcomputer under MATLAB.

The control of the three non-flat systems is based on high frequency control and approximations by averaged and flat systems (for other approaches, see, e.g., (Baillieul 1993, Bentsman 1987, Meerkov 1980)). We exploit here an idea due to the Russian physicist Kapitsa (Bogaevski and Povzner 1991, Landau and Lifshitz 1982) for stabilizing these three systems in the neighborhood of quite arbitrary positions and trajectories, and in particular positions which are not equilibrium points. This idea is closely related to a curiosity of classical mechanics that a double inverted pendulum (Stephenson 1908), and even the $N$ linked pendulums which are inverted and balanced on top of one another (Acheson 1993), 
can be stabilized in the same way. Closed-loop stabilization around reference averaged trajectories becomes straightforward by utilizing the endogenous feedback equivalence to linear controllable systems.

The paper is organized as follows. After some differential algebraic preliminaries, we define equivalence by endogenous feedback, flatness and defect. Their implications for uncontrolled dynamics and linear systems are examined. We discuss the link between flatness and controllability. In order to verify that some systems are not linearizable by dynamic feedback, we demonstrate a necessary condition of flatness, which is of geometric nature. The last two sections are devoted respectively to the flat and non-flat examples.

First drafts of various parts of this article have been presented in (Fliess et al. 1991, Fliess et al. 1992b, Fliess et al. 1992a, Fliess et al. 1993b, Fliess et al. 1993c).

\section{The algebraic framework}

We consider variables related by algebraic differential equations. This viewpoint, which possess a nice formalisation via differential algebra, is strongly related to Willems' behavioral approach (Willems 1991), where trajectories play a key role. We start with a brief review of differential fields (see also (Fliess 1990a, Fliess and Glad 1993)) and we refer to the books of Ritt (Ritt 1950) and Kolchin (Kolchin 1973) and Seidenberg's paper (Seidenberg 1952) for details. Basics on the customary (non-differential) field theory may be found in (Fliess 1990a, Fliess and Glad 1993) as well as in the textbook by Jacobson (Jacobson 1985) and Winter (Winter 1974) (see also (Fliess 1990a, Fliess and Glad 1993)); they will not be repeated here.

\subsection{Basics on differential fields}

An (ordinary) differential ring $R$ is a commutative ring equipped with a single derivation $\frac{d}{d t}={ }^{\bullet}$ such that

$$
\begin{aligned}
& \forall a \in R, \quad \dot{a}=\frac{d a}{d t} \in R \\
& \forall a, b \in R, \quad \frac{d}{d t}(a+b)=\dot{a}+\dot{b} \\
& \frac{d}{d t}(a b)=\dot{a} b+a \dot{b} .
\end{aligned}
$$

A constant $c \in R$ is an element such that $\dot{c}=0$. A ring of constants only contains constant elements. An (ordinary) differential field is an (ordinary) differential ring which is a field.

A differential field extension $L / K$ is given by two differential fields, $K$ and $L$, such that $K \subseteq L$ and such that the restriction to $K$ of the derivation of $L$ coincides with the derivation of $K$.

An element $\xi \in L$ is said to be differentially $K$-algebraic if, and only if, it satisfies an algebraic differential equation over $K$, i.e., if there exists a polynomial $\pi \in K\left[x_{0}, x_{1}, \ldots, x_{v}\right], \pi \neq 0$, such that $\pi\left(\xi, \dot{\xi}, \ldots, \xi^{(v)}\right)=0$. The extension $L / K$ is said to be differentially algebraic if, and only if, any element of $L$ is differentially $K$-algebraic. 
An element $\xi \in L$ is said to be differentially $K$-transcendental if, and only if, it is not differentially $K$-algebraic. The extension $L / K$ is said to be differentially transcendental if, and only if, there exists at least one element of $L$ that is differentially $K$-transcendental.

A set $\left\{\xi_{i} \mid i \in I\right\}$ of elements in $L$ is said to be differentially $K$-algebraically independent if, and only if, the set of derivatives of any order, $\left\{\xi_{i}^{(v)} \mid i \in I, v=0,1,2, \ldots\right\}$, is $K$-algebraically independent. Such an independent set which is maximal with respect to inclusion is called a differential transcendence basis of $L / K$. Two such bases have the same cardinality, i.e., the same number of elements, which is called the differential transcendence degree of $L / K$ : it is denoted by $\operatorname{diff} \operatorname{tr}^{0} L / K$. Notice that $L / K$ is differentially algebraic if, and only if, diff $\operatorname{tr}^{0} L / K=0$.

Theorem 1 For a finitely generated differential extension $L / K$, the next two properties are equivalent:

(i) $L / K$ is differentially algebraic;

(ii) the (non-differential) transcendence degree of $L / K$ is finite, i.e., $\operatorname{tr} d^{0} L / K<\infty$.

More details and some examples may be found in (Fliess and Glad 1993).

\subsection{Systems $^{2}$}

Let $k$ be a given differential ground field. A system is a finitely generated differential extension $\mathcal{D} / k^{3}$. Such a definition corresponds to a finite number of quantities which are related by a finite number of algebraic differential equations over $k^{4}$. We do not distinguish in this setting between input, state, output and other types of variables. This field-theoretic language therefore fits Willems standpoint (Willems 1991) on systems. The differential order of the system $\mathcal{D} / k$ is the differential transcendence degree of the extension $\mathcal{D} / k$.

Example Set $k=\mathbb{R} ; \mathcal{D} / k$ is the differential field generated by the four unknowns $x_{1}, x_{2}, x_{3}, x_{4}$ related by the two algebraic differential equations:

$$
\dot{x}_{1}+\ddot{x}_{3} \dot{x}_{4}=0, \quad \dot{x}_{2}+\left(x_{1}+\ddot{x}_{3} x_{4}\right) x_{4}=0 .
$$

Clearly, diff $\operatorname{tr}^{0} \mathcal{D} / k=2$ : it is equal to the number of unknowns minus the number of equations.

Denote by $k<u>$ the differential field generated by $k$ and by a finite set $u=\left(u_{1}, \ldots, u_{m}\right)$ of differential $k$-indeterminates: $u_{1}, \ldots, u_{m}$ are differentially $k$-algebraically independent, i.e.,

\footnotetext{
${ }^{2}$ See also (Fliess 1990a, Fliess and Glad 1993).

${ }^{3}$ Two systems $\mathcal{D} / k$ and $\tilde{\mathcal{D}} / k$ are, of course, identified if, and only if, there exists a differential $k$-isomorphism between them (a differential $k$-isomorphism commutes with $d / d t$ and preserves every element of $k$ ).

${ }^{4}$ It is a standard fact in classic commutative algebra and algebraic geometry (c.f. (Hartshorne 1977)) that one needs prime ideals for interpreting "concrete" equations in the language of field theory. In our differential setting, we of course need differential prime ideals (see (Kolchin 1973) and also (Fliess and Glad 1993) for an elementary exposition). The verification of the prime character of the differential ideals corresponding to all our examples is done in appendix A.
} 
diff $\operatorname{tr}^{0} k<u>/ k=m$. A dynamics with (independent) input $u$ is a finitely generated differentially algebraic extension $\mathcal{D} / k\langle u\rangle$. Note that the number $m$ of independent input channels is equal to the differential order of the corresponding system $\mathcal{D} / k$. An output $y=\left(y_{1}, \ldots, y_{p}\right)$ is a finite set of differential quantities in $\mathcal{D}$.

According to theorem 1, there exists a finite transcendence basis $x=\left(x_{1}, \ldots, x_{n}\right)$ of $\mathcal{D} / k<u>$. Consequently, any component of $\dot{x}=\left(\dot{x}_{1}, \ldots, \dot{x}_{n}\right)$ and of $y$ is $k<u>$-algebraically dependent on $x$, which plays the role of $a$ (generalized) state. This yields:

$$
\left\{\begin{aligned}
A_{1}\left(\dot{x}_{1}, x, u, \dot{u}, \ldots, u^{\left(\alpha_{1}\right)}\right) & =0 \\
& \vdots \\
A_{n}\left(\dot{x}_{n}, x, u, \dot{u}, \ldots, u^{\left(\alpha_{n}\right)}\right) & =0 \\
B_{1}\left(y_{1}, x, u, \dot{u}, \ldots, u^{\left(\beta_{1}\right)}\right) & =0 \\
& \vdots \\
B_{p}\left(y_{p}, x, u, \dot{u}, \ldots, u^{\left(\beta_{p}\right)}\right) & =0
\end{aligned}\right.
$$

where the $A_{i}$ 's and $B_{j}$ 's are polynomial over $k$. The integer $n$ is the dimension of the dynamics $\mathcal{D} / k<u>$. We refer to (Fliess and Hasler 1990, Fliess et al. 1993a) for a discussion of such generalized state-variable representations (8) and their relevance to practice.

Example (continued) Set $u_{1}=x_{3}$ and $u_{2}=\dot{x}_{4}$. The extension $\mathcal{D} / \mathbb{R}<u>$ is differentially algebraic and yields the representation

$$
\left\{\begin{array}{l}
\dot{x}_{1}=-\ddot{u}_{1} u_{2} \\
\dot{x}_{2}=-\left(x_{1}+\ddot{u}_{1} x_{4}\right) x_{4} \\
\dot{x}_{4}=u_{2} .
\end{array}\right.
$$

The dimension of the dynamics is 3 and $\left(x_{1}, x_{2}, x_{4}\right)$ is a generalized state. It would be 5 if we set $u_{1}=\ddot{x}_{3}$ and $u_{2}=\dot{x}_{4}$, and the corresponding representation becomes causal in the classical sense.

Remark 1 Take the dynamics $\mathcal{D} / k<u>$ and a finitely generated algebraic extension $\overline{\mathcal{D}} / \mathcal{D}$. The two dynamics $\mathcal{D} / k<u>$ and $\overline{\mathcal{D}} / k<u>$, which are of course equivalent, have the same dimension and can be given the same state variable representation (11). In the sequel, a system $\mathcal{D} / k<u>$ will be defined up to a finitely generated algebraic extension of $\mathcal{D}$.

\subsection{Modules and linear systems ${ }^{5}$}

Differential fields are to general for linear systems which are specified by linear differential equations. They are thus replaced by the following appropriate modules.

\footnotetext{
${ }^{5}$ See also (Fliess 1990b).
} 
Let $k$ be again a given differential ground field. Denote by $k\left[\frac{d}{d t}\right]$ the ring of linear differential operators of the type

$$
\sum_{\text {finite }} a_{\alpha} \frac{d^{\alpha}}{d t^{\alpha}} \quad\left(a_{\alpha} \in k\right) .
$$

This ring is commutative if, and only if, $k$ is a field of constants. Nevertheless, in the general non-commutative case, $k\left[\frac{d}{d t}\right]$ still is a principal ideal ring and the most important properties of left $k\left[\frac{d}{d t}\right]$-modules mimic those of modules over commutative principal ideal rings (see (Cohn 1985)).

Let $M$ be a left $k\left[\frac{d}{d t}\right]$ - module. An element $m \in M$ is said to be torsion if, and only if, there exists $\pi \in k\left[\frac{d}{d t}\right], \pi \neq 0$, such that $\pi \cdot m=0$. The set of all torsion elements of $M$ is a submodule $T$, which is called the torsion submodule of $M$. The module $M$ is said to be torsion if, and only if, $M=T$. The following result can regarded as the linear counterpart of theorem 1.

Proposition 1 For a finitely generated left $k\left[\frac{d}{d t}\right]$-module $M$, the next two properties are equivalent:

(i) $M$ is torsion;

(ii) the dimension of $M$ as a k-vector space is finite.

A finitely generated module $M$ is free if, and only if, its torsion submodule $T$ is trivial, i.e., $T=\{0\}^{6}$. Any finitely generated module $M$ can be written $M=T \oplus \Phi$ where $T$ is the torsion submodule of $M$ and $\Phi$ is a free module. The rank of $M$, denoted by $\mathrm{rk} M$, is the cardinality of any basis of $\Phi$. Thus, $M$ is torsion if, and only if, $\operatorname{rk} M=0$.

A linear system is, by definition, a finitely generated left $k\left[\frac{d}{d t}\right]$-module $\Lambda$. We are thus dealing with a finite number of variables which are related by a finite number of linear homogeneous differential equations and our setting appears to be strongly related to Willems' approach (Willems 1991). The differential order of $\Lambda$ is the rank of $\Lambda$.

A linear dynamics with input $u=\left(u_{1}, \ldots, u_{m}\right)$ is a linear system $\Lambda$ which contains $u$ such that the quotient module $\Lambda /[u]$ is torsion, where $[u]$ denotes the left $k\left[\frac{d}{d t}\right]$-module spanned by the components of $u$. The input is assumed to be independent, i.e., the module $[u]$ is free. This implies that the differential order of $\Lambda$ is equal to $m$. A classical Kalman state variable representation is always possible:

$$
\frac{d}{d t}\left(\begin{array}{c}
x_{1} \\
\vdots \\
x_{n}
\end{array}\right)=A\left(\begin{array}{c}
x_{1} \\
\vdots \\
x_{n}
\end{array}\right)+B\left(\begin{array}{c}
u_{1} \\
\vdots \\
u_{m}
\end{array}\right)
$$

where

- the dimension $n$ of the state $x=\left(x_{1}, \ldots, x_{n}\right)$, which is called the dimension of the dynamics, is equal to the dimension of the torsion module $\Lambda /[u]$ as a $k$-vector space.

\footnotetext{
${ }^{6}$ This is not the usual definition of free modules, but a characterization which holds for finitely generated modules over principal ideal rings, where any torsion-free module is free (see (Cohn 1985)).
} 
- the matrices $A$ and $B$, of appropriate sizes, have their entries in $k$.

An output $y=\left(y_{1}, \ldots, y_{p}\right)$ is a set of elements in $\Lambda$. It leads to the following output map:

$$
\left(\begin{array}{c}
y_{1} \\
\vdots \\
y_{p}
\end{array}\right)=C\left(\begin{array}{c}
x_{1} \\
\vdots \\
x_{n}
\end{array}\right)+\sum_{\text {finite }} D_{v} \frac{d^{v}}{d t^{v}}\left(\begin{array}{c}
u_{1} \\
\vdots \\
u_{m}
\end{array}\right) .
$$

The controllability of (10) can be expressed in a module-theoretical language which is independent of any denomination of variables. Controllability is equivalent to the freeness of the module $\Lambda$. This just is an algebraic counterpart (Fliess 1992) of Willems' trajectory characterization (Willems 1991). When the system is uncontrollable, the torsion submodule corresponds to the Kalman uncontrollability subspace.

Remark 2 The relationship with the general differential field setting is obtained by producing a formal multiplication. The symmetric tensor product (Jacobson 1985) of a linear system $\Lambda$, where $\Lambda$ is viewed as a $k$-vector space, is an integral differential ring. Its quotient field $\mathcal{D}$, which is a differential field, corresponds to the nonlinear field theoretic description of linear systems.

\subsection{Differentials and tangent linear systems}

Differential calculus, which plays such a role in analysis and in differential geometry, admits a nice analogue in commutative algebra (Kolchin 1973, Winter 1974), which has been extended to differential algebra by Johnson (Johnson 1969).

To a finitely generated differential extension $L / K$, associate a mapping $d_{L / K}: L \rightarrow \Omega_{L / K}$, called (Kähler) differential ${ }^{7}$ and where $\Omega_{L / K}$ is a finitely generated left $L\left[\frac{d}{d t}\right]$-module, such that

$$
\begin{array}{ll}
\forall a \in L & d_{L / K}\left(\frac{d a}{d t}\right)=\frac{d}{d t}\left(d_{L / K} a\right) \\
\forall a, b \in L & d_{L / K}(a+b)=d_{L / K} a+d_{L / K} b \\
\forall c \in K & d_{L / K}(a b)=b d_{L / K} a+a d_{L / K} b \\
d_{L / K} c=0 .
\end{array}
$$

Elements of $K$ behave like constants with respect to $d_{L / K}$. Properties of the extension $L / K$ can be translated into the linear module-theoretic framework of $\Omega_{L / K}$ :

- A set $\xi=\left(\xi_{1}, \ldots, \xi_{m}\right)$ is a differential transcendence basis of $L / K$ if, and only if, $d_{L / K} \xi=$ $\left(d_{L / K} \xi_{1}, \ldots, d_{L / K} \xi_{m}\right)$ is a maximal set of $L\left[\frac{d}{d t}\right]$-linearly independent elements in $\Omega_{L / K}$. Thus, $\operatorname{diff} \operatorname{tr} \mathrm{d}^{0} L / K=\operatorname{rk} \Omega_{L / K}$.

\footnotetext{
${ }^{7}$ For any $a \in L, d_{L / K} a$ should be intuitively understood, like in analysis and differential geometry, as a "small" variation of $a$.
} 
- The extension $L / K$ is differentially algebraic if, and only if, the module $\Omega_{L / K}$ is torsion. A set $x=\left(x_{1}, \ldots, x_{n}\right)$ is a transcendence basis of $L / K$ if, and only if, $d_{L / K} x=\left(d_{L / K} x_{1}, \ldots, d_{L / K} x_{n}\right)$ is a basis of $\Omega_{L / K}$ as $L$-vector space.

- The extension $L / K$ is algebraic if, and only if, $\Omega_{L / K}$ is trivial, i.e., $\Omega_{L / K}=\{0\}$.

The tangent (or variational) linear system associated to the system $\mathcal{D} / k$ is the left $\mathcal{D}\left[\frac{d}{d t}\right]$-module $\Omega_{\mathcal{D} / k}$. To a dynamics $\mathcal{D} / k<u>$ is associated the tangent (or variational) dynamics $\Omega_{\mathcal{D} / k}$ with the tangent (or variational) input $d_{L / K} u=\left(d_{L / K} u_{1}, \ldots, d_{L / K} u_{m}\right)$. The tangent (or variational) output associated to $y=\left(y_{1}, \ldots, y_{p}\right)$ is $d_{L / K} y=\left(d_{L / K} y_{1}, \ldots, d_{L / K} y_{p}\right)$.

\section{Equivalence, flatness and defect}

\subsection{Equivalence of systems and endogenous feedback}

Two systems $\mathcal{D} / k$ and $\tilde{\mathcal{D}} / k$ are said to be equivalent or equivalent by endogenous feedback if, and only if, any element of $\mathcal{D}$ (resp. $\tilde{\mathcal{D}}$ ) is algebraic over $\tilde{\mathcal{D}}$ (resp. $\mathcal{D})^{8}$. Two dynamics, $\mathcal{D} / k<u>$ and $\tilde{\mathcal{D}} / k<\tilde{u}>$, are said to be equivalent if, and only if, the corresponding systems, $\mathcal{D} / k$ and $\tilde{\mathcal{D}} / k$, are so.

Proposition 2 Two equivalent systems (resp. dynamics) possess the same differential order, i.e., the same number of independent input channels.

Proof Denote by $K$ the differential field generated by $\mathcal{D}$ and $\tilde{\mathcal{D}}: K / \mathcal{D}$ and $K / \tilde{\mathcal{D}}$ are algebraic extensions. Therefore,

$$
\operatorname{diff} \operatorname{tr} \mathrm{d}^{0} \mathcal{D} / k=\operatorname{diff} \operatorname{tr} \mathrm{d}^{0} K / k=\operatorname{diff} \operatorname{tr} \mathrm{d}^{0} \tilde{\mathcal{D}} / k
$$

Consider two equivalent dynamics, $\mathcal{D} / k<u>$ and $\tilde{\mathcal{D}} / k<\tilde{u}>$. Let $n$ (resp. $\tilde{n}$ ) be the dimension of $\mathcal{D} / k<u>$ (resp. $\tilde{\mathcal{D}} / k<\tilde{u}>$ ). In general, $n \neq \tilde{n}$. Write

$$
A_{i}\left(\dot{x}_{i}, x, u, \dot{u}, \ldots, u^{\left(\alpha_{i}\right)}\right)=0, \quad i=1, \ldots, n
$$

and

$$
\tilde{A}_{i}\left(\dot{\tilde{x}}_{i}, \tilde{x}, \tilde{u}, \dot{\tilde{u}}, \ldots, \tilde{u}^{\left(\tilde{\alpha}_{i}\right)}\right)=0, \quad i=1, \ldots, \tilde{n}
$$

the generalized state variable representations of $\mathcal{D} / k<u>$ and $\tilde{\mathcal{D}} / k<\tilde{u}>$, respectively. The algebraicity of any element of $\mathcal{D}($ resp. $\tilde{\mathcal{D}})$ over $\tilde{\mathcal{D}}($ resp. $\mathcal{D})$ yields the following relationships

\footnotetext{
${ }^{8}$ According to footnote 3 , this definition of equivalence can also be read as follows: two systems $\mathcal{D} / k$ and $\tilde{\mathcal{D}} / k$ are equivalent if, and only if, there exist two differential extensions $\mathscr{D} / \mathcal{D}$ and $\bar{D} / \overline{\mathcal{D}}$ which are algebraic (in the usual sense), and a differential $k$-automorphism $\Phi$ between $D / k$ and $\bar{D} / k$.
} 
between (11) and (12):

$$
\left\{\begin{array}{rccc}
\varphi_{i}\left(u_{i}, \tilde{x}, \tilde{u}, \dot{\tilde{u}}, \ldots, \tilde{u}^{\left(v_{i}\right)}\right) & =0 & i=1, \ldots, m \\
\sigma_{\alpha}\left(x_{\alpha}, \tilde{x}, \tilde{u}, \tilde{\tilde{u}}, \ldots, \tilde{u}^{\left(\mu_{\alpha}\right)}\right) & =0 & \alpha=1, \ldots, n \\
\tilde{\varphi}_{i}\left(\tilde{u}_{i}, x, u, \dot{u}, \ldots, u^{\left(\tilde{v}_{i}\right)}\right) & = & 0 & i=1, \ldots, m \\
\tilde{\sigma}_{\alpha}\left(\tilde{x}_{\alpha}, x, u, \dot{u}, \ldots, u^{\left(\tilde{\mu}_{\alpha}\right)}\right) & = & 0 & \alpha=1, \ldots, \tilde{n}
\end{array}\right.
$$

where the $\varphi_{i}$ 's, $\sigma_{\alpha}$ 's, $\tilde{\varphi}_{i}$ 's and $\tilde{\sigma}_{\alpha}$ 's are polynomials over $k$.

The two dynamic feedbacks corresponding to (13) are called endogenous as they do not necessitate the introduction of any variable that is transcendental over $\mathcal{D}$ and $\tilde{\mathcal{D}}$ (see also (Martin 1992)). If we know $\tilde{x}$ (resp. $x$ ), we can calculate $u$ (resp. $\tilde{u}$ ) from $\tilde{u}$ (resp. $u$ ) without integrating any differential equation. The relationship with general dynamic feedbacks is given in appendix $B$.

Remark 3 The tangent linear systems (see subsection 2.4) of two equivalent systems are strongly related and, in fact, are "almost identical". Take two equivalent systems $\mathcal{D}_{1} / k$ and $\mathcal{D}_{2} / k$ and denote by $\mathcal{D}$ the smallest algebraic extension of $\mathcal{D}_{1}$ and $\mathcal{D}_{2}$. It is straightforward to check that the three left $\mathcal{D}\left[\frac{d}{d t}\right]-$ modules $\Omega_{\mathcal{D} / k}, \mathcal{D} \otimes_{\mathcal{D}_{1}} \Omega_{\mathcal{D}_{1 / k}}$ and $\mathcal{D} \otimes_{\mathcal{D}_{2}} \Omega_{\mathcal{D}_{2} / k}$ are isomorphic (see (Hartshorne 1977, Jacobson 1985)).

\subsection{Flatness and defect}

Like in the non-differential case, a differential extension $L / K$ is said to be purely differentially transcendental if, and only if, there exists a differential transcendence basis $\xi=\left\{\xi_{i} \mid i \in I\right\}$ of $L / K$ such that $L=K<\xi>$. A system $\mathcal{D} / k$ is called purely differentially transcendental if, and only if, the extension $\mathcal{D} / k$ is so.

A system $\mathcal{D} / k$ is called (differentially) flat if, and only if, it is equivalent to a purely differentially transcendental system $L / k$. A differential transcendence basis $y=\left(y_{1}, \ldots, y_{m}\right)$ of $L / k$ such that $L=k\langle y\rangle$ is called a linearizing or flat output of the system $\mathcal{D} / k$.

Example (continued) Let us prove that $y=\left(y_{1}, y_{2}\right)$ with

$$
y_{1}=x_{2}+\frac{\left(x_{1}+\ddot{x}_{3} x_{4}\right)^{2}}{2 x_{3}^{(3)}}, \quad y_{2}=x_{3} .
$$

is a linearizing output for (7). Set $\sigma=x_{1}+\ddot{x}_{3} x_{4}$. Differentiating $y_{1}=x_{2}+\sigma^{2} / 2 y_{2}^{(3)}$, we have, using (7), $\sigma^{2}=-\frac{2 \dot{y}_{1}\left(y_{2}^{(3)}\right)^{2}}{y_{2}^{(4)}}$. Thus $x_{2}=y_{1}-\frac{\sigma^{2}}{2 y_{2}^{(3)}}$ is an algebraic function of $\left(y_{1}, \dot{y}_{1}, y_{2}^{(3)}, y_{2}^{(4)}\right)$. Since $x_{4}=-\frac{\dot{x}_{2}}{\sigma}$ and $x_{1}=\sigma-\ddot{y}_{2} x_{4}, x_{4}$ and $x_{1}$ are algebraic functions of $\left(y_{1}, \dot{y}_{1}, \ddot{y}_{1}, \ddot{y}_{2}, y_{2}^{(3)}, y_{2}^{(4)}, y_{2}^{(5)}\right)$. Remark there exist many other linearizing outputs such as $\tilde{y}=\left(\tilde{y}_{1}, \tilde{y}_{2}\right)=\left(2 y_{1} y_{2}^{(3)}, y_{2}\right)$, the inverse transformation being $y=\left(\tilde{y}_{1} / 2 \tilde{y}_{2}^{(3)}, \tilde{y}_{2}\right)$. 
Take an arbitrary system $\mathcal{D} / k$ of differential order $m$. Among all the possible choices of sets $z=\left(z_{1}, \ldots, z_{m}\right)$ of $m$ differential $k$-indeterminates which are algebraic over $\mathcal{D}$, take one such that $\operatorname{trd}^{0} \mathcal{D}<z>/ k<z>$ is minimum, say $\delta$. This integer $\delta$ is called the defect of the system $\mathcal{D} / k$. The next result is obvious.

Proposition 3 A system $\mathcal{D} / k$ is flat if, and only if, its defect is zero.

Example The defect of the system generated by $x_{1}$ and $x_{2}$ satisfying $\dot{x}_{1}=x_{1}+\left(\dot{x}_{2}\right)^{3}$ is one. Its general solution cannot be expressed without the integration of, at least, one differential equation.

\subsection{Basic examples}

\subsubsection{Uncontrolled dynamical systems}

An uncontrolled dynamical system is, in our field-theoretic language (Fliess 1990a), a finitely generated differentially algebraic extension $\mathcal{D} / k$ : diff $\operatorname{tr} \mathrm{d}^{0} \mathcal{D} / k=0$ implies the non-existence of any differential $k$-indeterminate algebraic over $\mathcal{D}$. Thus, the defect of $\mathcal{D} / k$ is equal to $\operatorname{tr} \mathrm{d} \mathcal{D} / k$, i.e., to the dimension of the dynamical system $\mathcal{D} / k$, which corresponds to the state variable representation $A_{i}\left(\dot{x}_{i}, x\right)=0$, where $x=\left(x_{1}, \ldots, x_{n}\right)$ is a transcendence basis of $\mathcal{D} / k$. Flatness means that $\mathcal{D} / k$ is algebraic in the (non-differential) sense: the dynamics $\mathcal{D} / k$ is then said to be trivial.

\subsubsection{Linear systems}

The defect of $\Lambda$ is, by definition, the defect of its associated differential field extension $\mathcal{D} / k$ (see remark 2).

Theorem 2 The defect of a linear system is equal to the dimension of its torsion submodule, i.e., to the dimension of its Kalman uncontrollable subspace. A linear system is flat if, and only if, it is controllable.

Proof Take the decomposition $\Lambda=T \oplus \Phi$, of section 2.3, where $T$ is the torsion submodule and $\Phi$ a free module. A basis $b=\left(b_{1}, \ldots, b_{m}\right)$ of $\Phi$ plays the role of a linearizing output when $\Lambda$ is free: the system then is flat. When $T \neq\{0\}$, the differential field extension $\mathcal{T} / k$ generated by $T$ is differentially algebraic and its (non-differential) transcendence degree is equal to the dimension of $T$ as $k$-vector space. The conclusion follows at once.

Remark 4 The above arguments can be made more concrete by considering a linear dynamics over $\mathbb{R}$. If it is controllable, we may write it, up to a static feedback, in its Brunovsky canonical form:

$$
y^{\left(v_{i}\right)}=u_{i}, \quad(i=1, \ldots, m)
$$


where the $v_{i}$ 's are the controllability indices and $y=\left(y_{1}, \ldots, y_{m}\right)$ is a linearizing output. In the uncontrollable case, the defect $d$ is the dimension of the uncontrollable subspace:

$$
\frac{d}{d t}\left(\begin{array}{c}
\xi_{1} \\
\vdots \\
\xi_{d}
\end{array}\right)=M\left(\begin{array}{c}
\xi_{1} \\
\vdots \\
\xi_{d}
\end{array}\right)
$$

where $M$ is a $d \times d$ matrix over $\mathbb{R}$.

\subsection{A necessary condition for flatness}

Consider the system $\mathcal{D} / k$ where $\mathcal{D}=k<w>$ is generated by a finite set $w=\left(w_{1}, \ldots, w_{q}\right)$. The $w_{i}$ 's are related by a finite set, $\Xi\left(w, \dot{w}, \ldots, w^{(v)}\right)=0$, of algebraic differential equations. Define the algebraic variety $S$ corresponding to $\Xi\left(\xi^{0}, \ldots, \xi^{\nu}\right)=0$ in the $(v+1) q$-dimensional affine space with coordinates

$$
\xi^{j}=\left(\xi_{1}^{j}, \ldots, \xi_{q}^{j}\right), \quad j=0,1, \ldots, v .
$$

Theorem 3 If the system $\mathcal{D} / k$ is flat, the affine algebraic variety $S$ contains at each regular point a straight line parallel to the $\xi^{\nu}$-axes.

Proof The components of $w, \dot{w}, \ldots, w^{(\nu-1)}$ are algebraically dependent on the components of a linearizing output $y=\left(y_{1}, \ldots, y_{m}\right)$ and a finite number of their derivatives. Let $\mu$ be the highest order of these derivatives. The components of $w^{(\nu)}$ depend linearly on the components of $y^{(\mu+1)}$, which play the role of independent parameters for the coordinates $\xi_{1}^{v}, \ldots, \xi_{q}^{v}$.

The above condition is not sufficient. Consider the system $\mathcal{D} / \mathbb{R}$ generated by $\left(x_{1}, x_{2}, x_{3}\right)$ satisfying $\dot{x}_{1}=\left(\dot{x}_{2}\right)^{2}+\left(\dot{x}_{3}\right)^{3}$. This system does not satisfy the necessary condition: it is not flat. The same system $\mathcal{D}$ can be defined via the quantities $\left(x_{1}, x_{2}, x_{3}, x_{4}\right)$ related by $\dot{x}_{1}=\left(x_{4}\right)^{2}+\left(\dot{x}_{3}\right)^{3}$ and $x_{4}=\dot{x}_{2}$. Those new equations now satisfy our necessary criterion.

\subsection{Flatness and controllability}

Sussmann and Jurdjevic (Sussmann and Jurdjevic 1972) have introduced in the differential geometric setting the concept of strong accessibility for dynamics of the form $\dot{x}=f(x, u)$. Sontag (Sontag 1988) showed that strong accessibility implies the existence of controls such that the linearized system around a trajectory passing through a point $a$ of the state-space is controllable. Coron (Coron 1994) and Sontag (Sontag 1992) demonstrated that, for any $a$, those controls are generic.

The above considerations with those of section 2.3 and 2.4 lead in our context to the following definition of controllability, which is independent of any distinction between variables: a system $\mathcal{D} / k$ is said to be controllable (or strongly accessible) if, and only if, its tangent linear system is controllable, i.e., if, and only if, the module $\Omega_{\mathcal{D} / k}$ is free.

Remark 3 shows that this definition is invariant under our equivalence via endogenous feedback.

Proposition 4 A flat system is controllable 
Proof It suffices to prove it for a purely differentially transcendental extensions $k<y>/ k$, where $y=\left(y_{1}, \ldots, y_{m}\right)$. The module $\Omega_{k<y>/ k}$, which is spanned by $d_{k<y>/ k} y_{1}, \ldots, d_{k<y>/ k} y_{m}$, is necessarily free.

The converse is false as demonstrated by numerous examples of strongly accessible single-input dynamics $\dot{x}=f(x, u)$ which are not linearizable by static feedback and therefore neither by dynamic ones (Charlet et al. 1989).

Flatness which is equivalent to the possibility of expressing any element of the system as a function of the linearizing output and a finite number of its derivatives, may be viewed as the nonlinear extension of linear controllability, if the latter is characterized by free modules. Whereas the strong accessibility property only is an "infinitesimal" generalization of linear controllability, flatness should be viewed as a more "global" and, perhaps, as a more tractable one. This will be enhanced in section 5 where controllable systems of nonzero defect are treated using high-frequency control that enables to approximate them by flat systems for which the control design is straightforward.

\section{Examples and control of flat systems}

The verification of the prime character of the differential ideals corresponding to all our examples is done in appendix A. This means that the equations defining all our examples can be rigorously interpreted in the language of differential field theory.

\subsection{The 2-D crane}

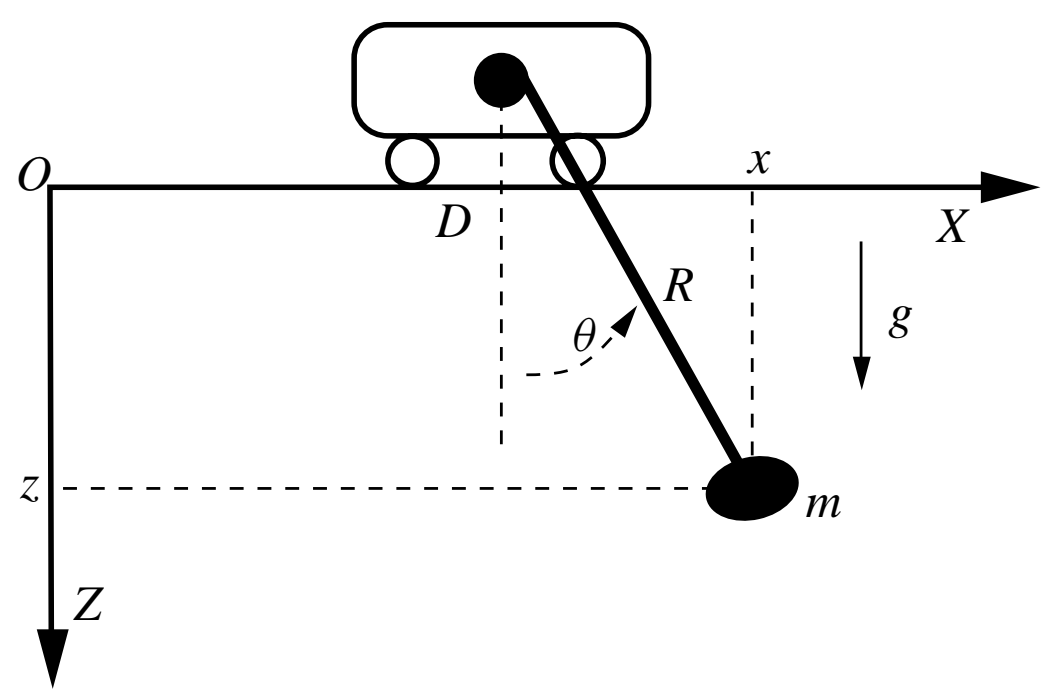

Figure 1: The two dimensional crane. 
Consider the crane displayed on figure 1 which is a classical object of control study (see, e.g., (D’Andréa-Novel and Lévine 1990), (Marttinen et al. 1990)). The dynamics can be divided into two parts. The first part corresponds to the motor drives and industrial controllers for trolley travels and rolling up and down the rope. The second part is relative to the trolley load, the behavior of which is very similar to the pendulum one. We concentrate here on the pendulum dynamics by assuming that

- the traversing and hoisting are control variables,

- the trolley load remains in a fixed vertical plane $O X Z$,

- the rope dynamics are negligible.

A dynamic model of the load can be derived by Lagrangian formalism. It can also be obtained, in a very simple way, by writing down all the differential (Newton law) and algebraic (geometric constraints) equations describing the pendulum behavior:

$$
\left\{\begin{aligned}
m \ddot{x} & =-T \sin \theta \\
m \ddot{z} & =-T \cos \theta+m g \\
x & =R \sin \theta+D \\
z & =R \cos \theta
\end{aligned}\right.
$$

where

- $(x, z)$ (the coordinates of the load $m$ ), $T$ (the tension of the rope) and $\theta$ (the angle between the rope and the vertical axis $O Z$ ) are the unknown variables;

- $D$ (the trolley position) and $R$ (the rope length) are the input variables.

From (14), it is clear that $\sin \theta, T, D$ and $R$ are algebraic functions of $(x, z)$ and their derivatives:

$$
\sin \theta=\frac{x-D}{R}, \quad T=\frac{m R(g-\ddot{z})}{z}, \quad(\ddot{z}-g)(x-D)=\ddot{x} z, \quad(x-D)^{2}+z^{2}=R^{2}
$$

that is

$$
\left\{\begin{aligned}
D & =x-\frac{\ddot{x} z}{\ddot{z}-g} \\
R^{2} & =z^{2}+\left(\frac{\ddot{x} z}{\ddot{z}-g}\right)^{2} .
\end{aligned}\right.
$$

Thus, system (14) is flat with $(x, z)$ as linearizing output.

Remark 5 Assume that the modeling equations (14) are completed with the following traversing and hoisting dynamics:

$$
\left\{\begin{aligned}
M \ddot{D} & =\mathcal{F}-\lambda \dot{D}+T \sin \theta \\
\frac{J}{\rho^{2}} \ddot{R} & =\mathcal{C}-\frac{\mu}{\rho} \dot{R}-T \rho
\end{aligned}\right.
$$


where the new variables $\mathcal{F}$ and $\mathcal{C}$ are, respectively the external force applied to the trolley and the hoisting torque. The other quantities $(M, J, \rho, \lambda, \mu)$ are constant physical parameters. Then $(14,16)$ is also flat with the same linearizing output $(x, z)$. This explains without any additional computation why the system considered in (D'Andréa-Novel and Lévine 1990) is linearizable via dynamic feedback.

Let us now address the following question which is one of the basic control problems for a crane: how can one carry a load $m$ from the steady-state $R=R_{1}>0$ and $D=D_{1}$ at time $t_{1}$, to the steady-state $R=R_{2}>0$ and $D=D_{2}$ at time $t_{2}>t_{1}$ ?

It is clear that any motion of the load induces oscillations that must be canceled at the end of the load transport. We propose here a very simple answer to this question when the crane can be described by (14). This answer just consists in using (15).

Consider a smooth curve $\left.\left[t_{1}, t_{2}\right] \ni t \rightarrow(\alpha(t), \gamma(t)) \in \mathbb{R} \times\right] 0,+\infty[$ such that

- for $i=1,2,\left(\alpha\left(t_{i}\right), \gamma\left(t_{i}\right)\right)=\left(D_{i}, R_{i}\right)$, and $\frac{d^{r}}{d t^{r}}(\alpha, \gamma)\left(t_{i}\right)=0$ with $r=1,2,3,4$.

- for all $t \in\left[t_{1}, t_{2}\right], \ddot{\gamma}(t)<g$.

Then the solution of (14) starting at time $t_{1}$ from the steady-state $D_{1}$ and $R_{1}$, and with the control trajectory defined, for $t \in\left[t_{1}, t_{2}\right]$, by

$$
\left\{\begin{array}{l}
D(t)=\alpha(t)-\frac{\ddot{\alpha}(t) \gamma(t)}{\ddot{\alpha}(t)-g} \\
R(t)=\sqrt{\gamma^{2}(t)+\left(\frac{\ddot{\alpha}(t) \gamma(t)}{\ddot{\gamma}(t)-g}\right)^{2}}
\end{array}\right.
$$

and, for $t>t_{2}$, by $(D(t), R(t))=\left(D_{2}, R_{2}\right)$, leads to a load trajectory $t \rightarrow(x(t), z(t))$ such that $(x(t), z(t))=(\alpha(t), \gamma(t))$ for $t \in\left[t_{1}, t_{2}\right]$ and $(x(t), z(t))=\left(D_{2}, R_{2}\right)$ for $t \geq t_{2}$. Notice that, since for all $t \in\left[t_{1}, t_{2}\right], \ddot{z}(t)<g$, the rope tension $T=\frac{m R(g-\ddot{z})}{z}$ remains always positive and the description of the system by (14) remains reasonable.

This results from the following facts. The generalized state variable description of the system is the following (Fliess et al. 1991, Fliess et al. 1993a):

$$
R \ddot{\theta}=-2 \dot{R} \dot{\theta}-\ddot{D} \cos \theta-g \sin \theta .
$$

Since $\alpha$ and $\gamma$ are smooth, $D$ and $R$ are at least twice continuously differentiable. Thus, the classical existence and uniqueness theorem ensures that the above ordinary differential equation admits a unique smooth solution that is nothing but $\theta(t)=\arctan (\alpha(t)-D(t)) / \gamma(t))$.

The approximation of the crane dynamics by (14) implies that the motor drives and industrial lowlevel controllers (trolley travels and rolling up and down the rope) produce fast and stable dynamics (see remark 5). Thus, if these dynamics are stable and fast enough, classical results of singular perturbation theory of ordinary differential equation (see, e.g., (Tikhonov et al. 1980)), imply that the control (17) leads to a final configuration close to the steady-state defined by $D_{2}$ and $R_{2}$. 
In the simulations displayed here below, we have verified that the addition of reasonable fast and stable regulator dynamics modifies only slightly the final position $\left(R_{2}, D_{2}\right)$. Classical proportionalintegral controller for $D$ and $R$ are added to (14). The typical regulator time constants are equal to one tenth of the period of small oscillations $\left(\frac{1}{10} 2 \pi \sqrt{R / g} \approx 0.3 \mathrm{~s}\right)$ (see (Fliess et al. 1991)).
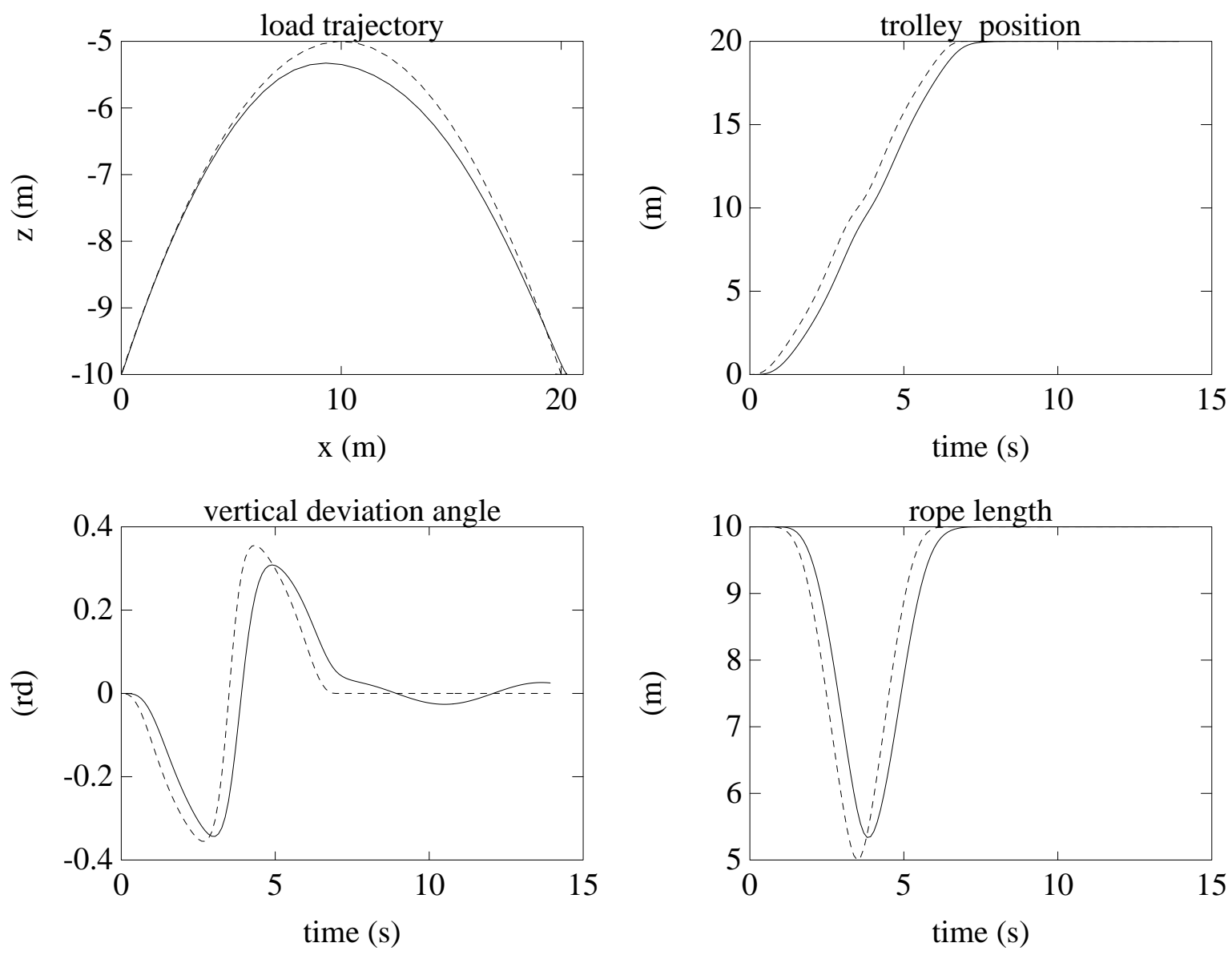

Figure 2: Simulation of the control defined by (17) without (solid lines) and with (dot lines) ideal low-level controllers for $D$ and $R$.

For the simulations presented in figure 2, the transport of the load $m$ may be considered as a rather fast one: the horizontal motion of $D$ is of $10 \mathrm{~m}$ in $3.5 \mathrm{~s}$; the vertical motion of $R$ is up to $5 \mathrm{~m}$ in $3.5 \mathrm{~s}$. Compared with the low-level regulator time constants $(0.1$ and $0.3 \mathrm{~s})$, such motions are not negligible. This explains the transient mismatch between the ideal and non-ideal cases. Nevertheless, the final control performances are not seriously altered: the residual oscillations of the load after $7 \mathrm{~s}$ admit less than $3 \mathrm{~cm}$ of horizontal amplitude. Such small residual oscillations can be canceled via a simple PID 
regulator with the vertical deviation $\theta$ as input and the set-point of $D$ as output.

The simulations illustrate the importance of the linearizing output $(x, z)$. When the regulations of $R$ and $D$ are suitably designed, it is possible to use the control given in (17) for fast transports of the load $m$ from one point to another. The simplicity and the independence of (17) with respect to the system parameters (except $g$ ) constitute its main practical interests.

Remark 6 Similar calculations can be performed when a second horizontal direction $\mathrm{OX}_{2}$, orthogonal to $O X_{1}=O X$, is considered. Denoting then by $\left(x_{1}, x_{2}, z\right)$ the cartesian coordinates of the load, $R$ the rope length and $\left(D_{1}, D_{2}\right)$ the trolley horizontal position, the system is described by

$$
\left\{\begin{aligned}
(\ddot{z}-g)\left(x_{1}-D_{1}\right) & =\ddot{x}_{1} z \\
(\ddot{z}-g)\left(x_{2}-D_{2}\right) & =\ddot{x}_{2} z \\
\left(x_{1}-D_{1}\right)^{2}+\left(x_{2}-D_{2}\right)^{2}+z^{2} & =R^{2} .
\end{aligned}\right.
$$

This system is clearly flat with the cartesian coordinates of the load, $\left(x_{1}, x_{2}, z\right)$, as flat output.

Remark 7 In (D'Andréa-Novel et al. 1992b), the control of a body of mass $m$ around a rotation axle $\Delta$ of constant direction is investigated. This system is flat as a consequence of the following considerations. According to an old result due to Huygens (see, e.g. (Whittaker 1937, p. 131-132)), the equations describing the motion are equivalent to those of a pendulum of the same mass $m$ and of length $l=\frac{J}{m d}$ where $d \neq 0$ is the vertical distance between the mass center $G$ and the axle $\Delta, J$ is the inertial moment around $\Delta$. Denoting by $u$ and $v$, respectively, the vertical and horizontal positions of $\Delta$, the equations of motion are the following (compare to (15)):

$$
\left\{\begin{array}{l}
\frac{\ddot{u}}{u-x}=\frac{\ddot{v}-g}{v-z} \\
(u-x)^{2}+(v-z)^{2}=l^{2}
\end{array}\right.
$$

where $(x, z)$ are the horizontal and vertical coordinates of the Huygens oscillation center. Clearly $(x, z)$ is a linearizing output.

Remark 8 The examples corresponding to the crane, Huygens' oscillation center (see remark 7) and the car with $n$-trailers here below, illustrate the fact that linearizing outputs admit most often a clear physical interpretation.

\subsection{The car with $n$-trailers}

\subsubsection{Modeling equations}

Steering a car with $n$ trailers is now the object of active researches (Laumond 1991, Murray and Sastry 1993, Monaco and Normand-Cyrot 1992, Rouchon et al. 1993a, Tilbury et al. 1993). The flatness of a basic model $^{9}$ of this system combined with the use of Frénet formula lead to a complete and simple solution

\footnotetext{
${ }^{9}$ More realistic models where trailer $i$ is not directly hitched to the center of the axle of trailer $i-1$ are considered in (Martin and Rouchon 1993, Rouchon et al. 1993b).
} 


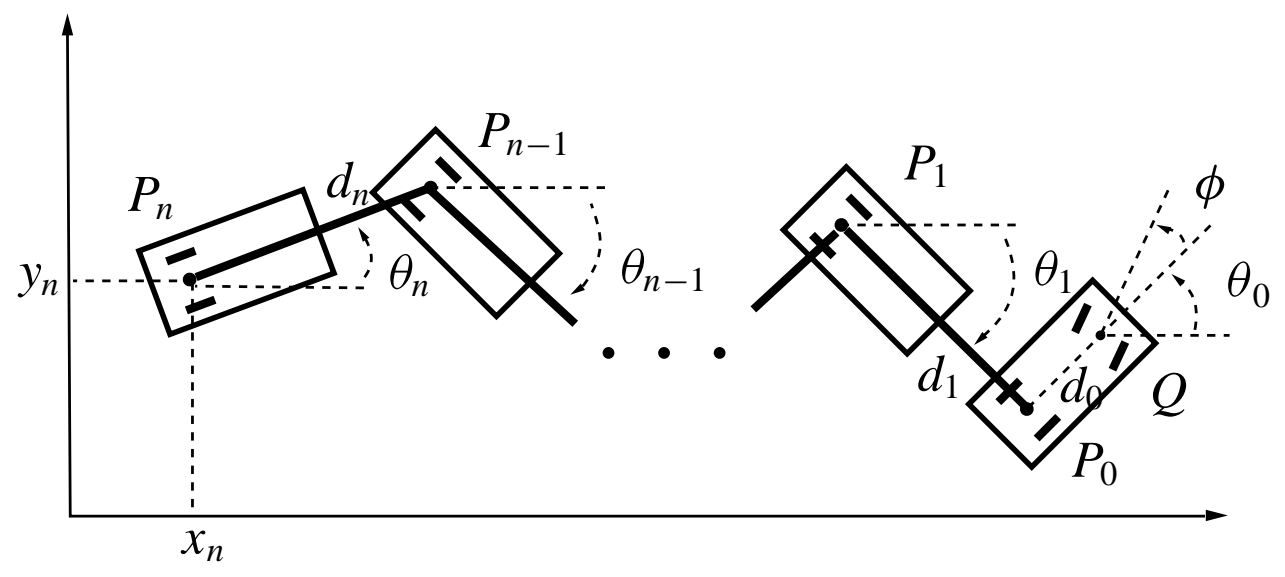

Figure 3: The kinematic car with $n$ trailers.

of the motion planning problem without obstacles. Notice that most of nonholonomic mobile robots are flat (D'Andréa-Novel et al. 1992a, Campion et al. 1992).

The hitch of trailer $i$ is attached to the center of the rear axle of trailer $i-1$. The wheels are aligned with the body of the trailer. The two control inputs are the driving velocity (of the rear wheels of the car) and the steering velocity (of the front wheels of the car). The constraints are based on allowing the wheels to roll and spin without slipping. For the steering front wheels of the car, the derivation is simplified by assuming them as a single wheel at the midpoint of the axle. The resulting dynamics are described by the following equations (the notations are those of (Murray and Sastry 1993) and summarized on figure 3):

$$
\left\{\begin{aligned}
\dot{x}_{0} & =u_{1} \cos \theta_{0} \\
\dot{y}_{0} & =u_{1} \sin \theta_{0} \\
\dot{\phi} & =u_{2} \\
\dot{\theta}_{0} & =\frac{u_{1}}{d_{0}} \tan \phi \\
\dot{\theta}_{i} & =\frac{u_{1}}{d_{i}}\left(\prod_{j=1}^{i-1} \cos \left(\theta_{j-1}-\theta_{j}\right)\right) \sin \left(\theta_{i-1}-\theta_{i}\right) \quad \text { for } i=1, \ldots, n
\end{aligned}\right.
$$

where $\left.\left(x_{0}, y_{0}, \phi, \theta_{0}, \ldots, \theta_{n}\right) \in \mathbb{R}^{2} \times\right]-\pi / 2,+\pi / 2\left[\times\left(S^{1}\right)^{n+1}\right.$ is the state, $\left(u_{1}, u_{2}\right)$ is the control and $d_{0}, d_{1}, \ldots, d_{n}$ are positive parameters (lengths). As displayed on figure 3 , we denote by $P_{i}$, the medium point of the wheel axle of trailer $i$, for $i=1, \ldots, n$. The medium point of the rear (resp. front) wheel axle of the car is denoted by $P_{0}$ (resp. $Q$ ). 


\subsubsection{Cartesian coordinates of $P_{n}$ as flat output}

Denote by $\left(x_{i}, y_{i}\right)$ the cartesian coordinates of $P_{i}, i=0,1, \ldots, n$ :

$$
\begin{aligned}
& x_{i}=x_{0}-\sum_{j=1}^{i} d_{j} \cos \theta_{j} \\
& y_{i}=y_{0}-\sum_{j=1}^{i} d_{j} \sin \theta_{j} .
\end{aligned}
$$

A direct computation shows that $\tan \theta_{i}=\frac{\dot{y}_{i}}{\dot{x}_{i}}$. Since, for $i=0, \ldots, n-1, x_{i}=x_{i+1}+d_{i+1} \cos \theta_{i+1}$ and $y_{i}=y_{i+1}+d_{i+1} \sin \theta_{i+1}$, the variables $\theta_{n}, x_{n-1}, y_{n-1}, \theta_{n-1}, \ldots, \theta_{1}, x_{0}, y_{0}$ and $\theta_{0}$ are functions of $x_{n}$ and $y_{n}$ and their derivatives up to the order $n+1$. But $u_{1}=\dot{x}_{0} / \cos \theta_{0}, \tan \phi=d_{0} \dot{\theta}_{0} / u_{1}$ and $u_{2}=\dot{\phi}$. Thus, the entire state and the control are functions of $x_{n}$ and $y_{n}$ and their derivatives up to order $n+3$.

This proves that the car with $n$ trailers described by (18) is a flat system: the linearizing output corresponds to the cartesian coordinates of the point $P_{n}$, the medium point of the wheel axle of the last trailer.

Flatness implies that for generic values of the state, the strong accessibility rank associated to the control system (18) is maximum and equal to its state-space dimension: the system is thus controllable.

The singularity which might occur when dividing by $\dot{x}_{i}=0$ in $\tan \theta_{i}=\dot{y}_{i} / \dot{x}_{i}$, can be avoided by the following developments.

\subsubsection{Motion planing using flatness}

In (Rouchon et al. 1993a, Rouchon et al. 1993b), the following result was sketched.

Proposition 5 Consider (18) and two different state-space configurations: $\tilde{p}=\left(\tilde{x}_{0}, \tilde{y}_{0}, \tilde{\phi}, \tilde{\theta}_{0}, \ldots, \tilde{\theta}_{n}\right)$ and $\bar{p}=\left(\bar{x}_{0}, \bar{y}_{0}, \bar{\phi}, \bar{\theta}_{0}, \ldots, \bar{\theta}_{n}\right)$. Assume that the angles $\tilde{\theta}_{i-1}-\tilde{\theta}_{i}, i=1, \ldots, n, \tilde{\phi}, \bar{\theta}_{i-1}-\bar{\theta}_{i}$, $i=1, \ldots, n$, and $\bar{\phi}$ belong to ] $-\pi / 2, \pi / 2$ [. Then, there exists a smooth open-loop control $[0, T] \ni$ $t \rightarrow\left(u_{1}(t), u_{2}(t)\right)$ steering the system from $\tilde{p}$ at time 0 to $\bar{p}$ at time $T>0$, such that the angles $\theta_{i-1}-\theta_{i}$, $i=1, \ldots, n$, and $\phi(i=1, \ldots, n)$ always remain in $]-\pi / 2, \pi / 2\left[\right.$ and such that $\left(u_{1}(t), u_{2}(t)\right)=0$ for $t=0, T$.

The conditions $\left.\theta_{i-1}-\theta_{i} \in\right]-\pi / 2, \pi / 2[(i=1, \ldots, n)$ and $\phi \in]-\pi / 2, \pi / 2[$ are meant for avoiding some undesirable geometric configurations: trailer $i$ should not be in front of trailer $i-1$.

The detailed proof is given in the appendix and relies basically on the fact that the system is flat. It is constructive and gives explicitly $\left(u_{1}(t), u_{2}(t)\right)$. The involved computations are greatly simplified by a simple geometric interpretation of the rolling without slipping conditions and the use of the Frénet formula. Here, we just recall this geometric construction and give the explicit formula for parking a car with two trailers. The Frénet formula are recalled in the appendix.

Denote by $\mathfrak{C}_{i}$ the curve followed by $P_{i}, i=0, \ldots, n$. As displayed on figure 4 , the point $P_{i-1}$ belongs to the tangent to $\mathcal{C}_{i}$ at $P_{i}$ and at the fixed distance $d_{i}$ from $P_{i}$ :

$$
P_{i-1}=P_{i}+d_{i} \tau_{i}
$$




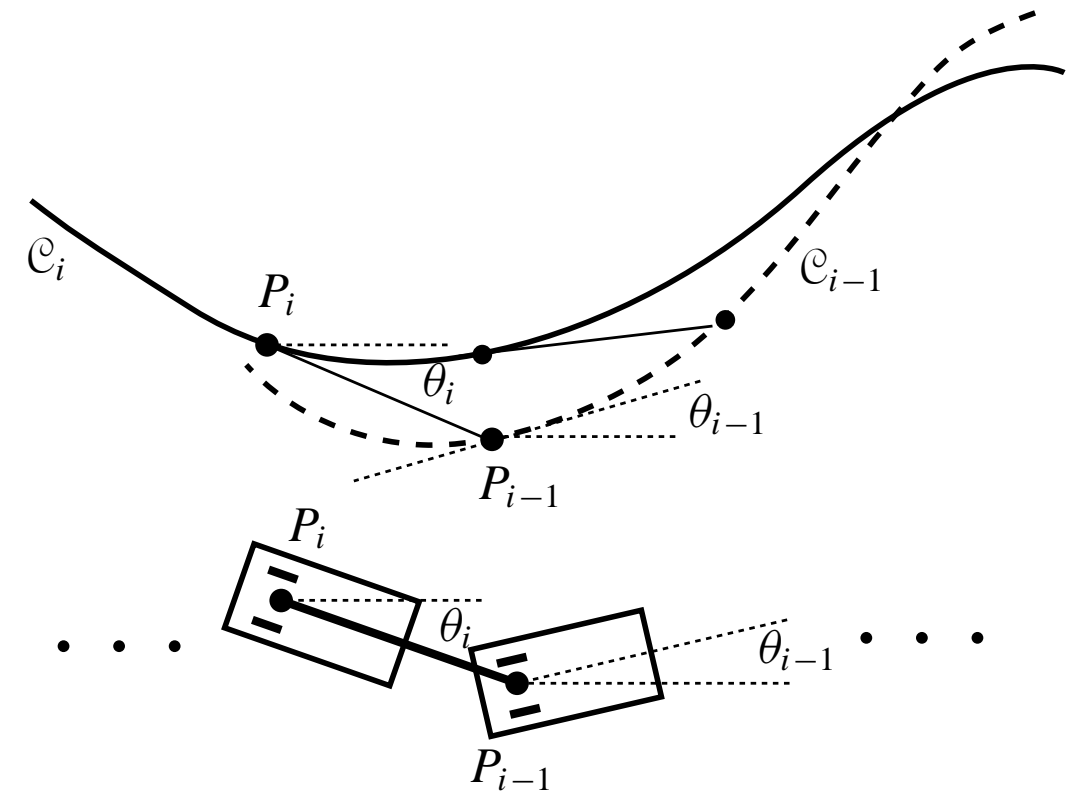

Figure 4: The geometric interpretation of the rolling without slipping conditions.

with $\tau_{i}$ the unitary tangent vector to $\mathfrak{C}_{i}$. Differentiating this relation with respect to $s_{i}$, the arc length of $\mathfrak{C}_{i}$, leads to

$$
\frac{d}{d s_{i}} P_{i-1}=\tau_{i}+d_{i} \kappa_{i} v_{i}
$$

where $v_{i}$ is the unitary vector orthogonal to $\tau_{i}$ and $\kappa_{i}$ is the curvature of $\mathcal{C}_{i}$. Since $\frac{d}{d s_{i}} P_{i-1}$ gives the tangent direction to $\mathcal{C}_{i-1}$, we have

$$
\tan \left(\theta_{i-1}-\theta_{i}\right)=d_{i} \kappa_{i}
$$

\subsubsection{Parking simulations of the 2-trailer system}

We now restrict to the particular case $n=2$. We show how the previous analysis can be employed to solve the parking problem. The simulations of figures 5 and 6 have been written in MATLAB. They can be obtained upon request from the fourth author via electronic mail (rouchon@ cas.ensmp.fr).

The car and its trailers are initially in $A$ with angles $\theta_{2}=\theta_{1}=\theta_{0}=\pi / 6, \phi=0$. The objective is to steer the system to $C$ with final angles $\left(\theta_{2}, \theta_{1}, \theta_{0}, \phi\right)=0$. We consider the two smooth curves $\mathcal{C}_{A B}$ and $\mathcal{C}_{C B}$ of the figure 5 , defined by their natural parameterizations $\left[0, L_{A B}\right] \ni s \rightarrow P_{A B}(s)$ and $\left[0, L_{C B}\right] \ni s \rightarrow P_{C B}(s)$, respectively $\left(P_{A B}(0)=A, P_{C B}(0)=C, L_{A B}\right.$ is the length of $\mathcal{C}_{A B}$ and $L_{C B}$ the length of $\left.\mathcal{C}_{C B}\right)$. Their curvatures are denoted by $\kappa_{A B}(s)$ and $\kappa_{C B}(s)$. These curves shall be followed by $P_{2}$. The initial and final system configuration in $A$ and $C$ impose $\kappa_{A B}(0)=\frac{d}{d s} \kappa_{A B}(0)=\frac{d^{2}}{d s^{2}} \kappa_{A B}(0)=0$ and $\kappa_{C B}(0)=\frac{d}{d s} \kappa_{C B}(0)=\frac{d^{2}}{d s^{2}} \kappa_{C B}(0)=0$. We impose additionally that $A B$ and $C B$ are tangent at $B$ 


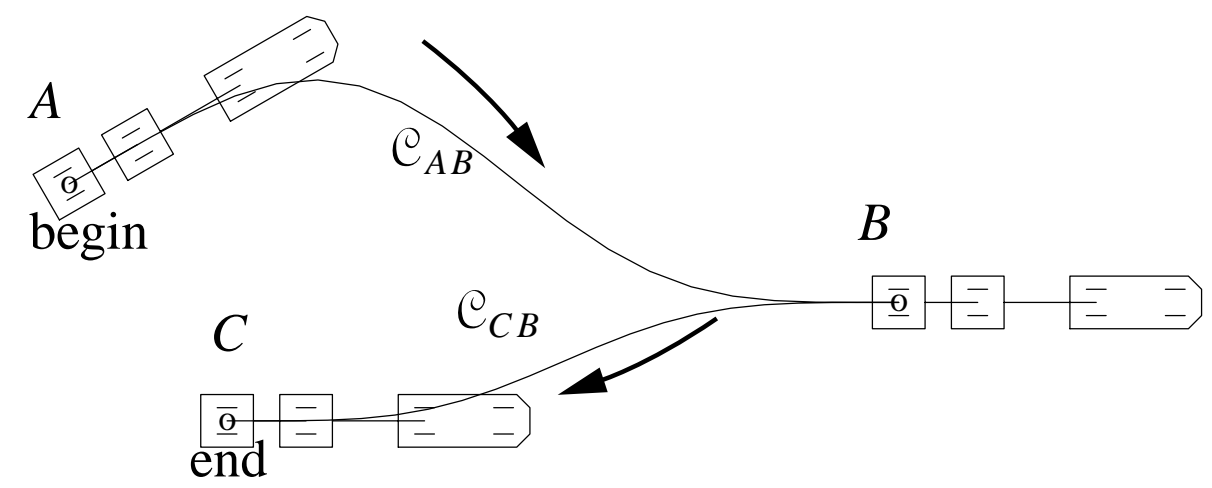

Figure 5: parking the car with two trailers from $A$ to $B$ via $C$.

and

$$
\kappa_{A B}\left(L_{A B}\right)=\frac{d}{d s} \kappa_{A B}\left(L_{A B}\right)=\frac{d^{2}}{d s^{2}} \kappa_{A B}\left(L_{A B}\right)=\kappa_{C B}\left(L_{C B}\right)=\frac{d}{d s} \kappa_{C B}\left(L_{C B}\right)=\frac{d^{2}}{d s^{2}} \kappa_{C B}\left(L_{C B}\right)=0 .
$$

It is straightforward to find curves satisfying such conditions. For the simulation of figure 6, we take polynomial curves of degree 9.

Proposition 5 implies that, if $P_{2}$ follows $\mathcal{C}_{A B}$ and $\mathcal{C}_{C B}$ as displayed on figure 5, then the initial and final states will be as desired. Take a smooth function $[0, T] \ni t \rightarrow s(t) \in\left[0, L^{A B}\right]$ such that $s(0)=0$, $s(T)=L^{A B}$ and $\dot{s}(0)=\dot{s}(T)=0$. This leads to smooth control trajectories $[0, T] \ni t \rightarrow u_{1}(t) \geq 0$ and $[0, T] \ni t \rightarrow u_{2}(t)$ steering the system from $A$ at time $t=0$ to $B$ at time $t=T$. Similarly, $[T, 2 T] \ni t \rightarrow s(t) \in\left[0, L^{C B}\right]$ such that $s(T)=L^{C B}, s(2 T)=0$ and $\dot{s}(T)=\dot{s}(2 T)=0$ leads to control trajectories $[T, 2 T] \ni t \rightarrow u_{1}(t) \leq 0$ and $[T, 2 T] \ni t \rightarrow u_{2}(t)$ steering the system from $B$ to $C$. This gives the motions displayed on figure 6 with forwards motions from $A$ to $B$, backwards motions from $B$ to $C$ and a stop in $B$.

Let us detail the calculation of the control trajectories for the motion from $A$ to $B$. Similar calculations can be done for the motion from $B$ to $C$. The curve $\mathcal{C}_{A B}$ corresponds to the curve $\mathcal{C}_{i}$ of figure 4 with $i=2$. Assume that $\mathfrak{C}_{A B}$ is given via the regular parameterization, $y=f(x)((x, y)$ are the cartesian coordinates and $f$ is a polynomial of degree 9$)$. Denote by $s_{i}$ the arc length of curve $\mathfrak{C}_{i}$, $i=0,1,2$. Then $d s_{2}=\sqrt{1+(d f / d x)^{2}} d x$ and the curvature of $\mathfrak{C}_{2}$ is given by

$$
\kappa_{2}=\frac{d^{2} f / d x^{2}}{\left(1+(d f / d x)^{2}\right)^{3 / 2}} .
$$




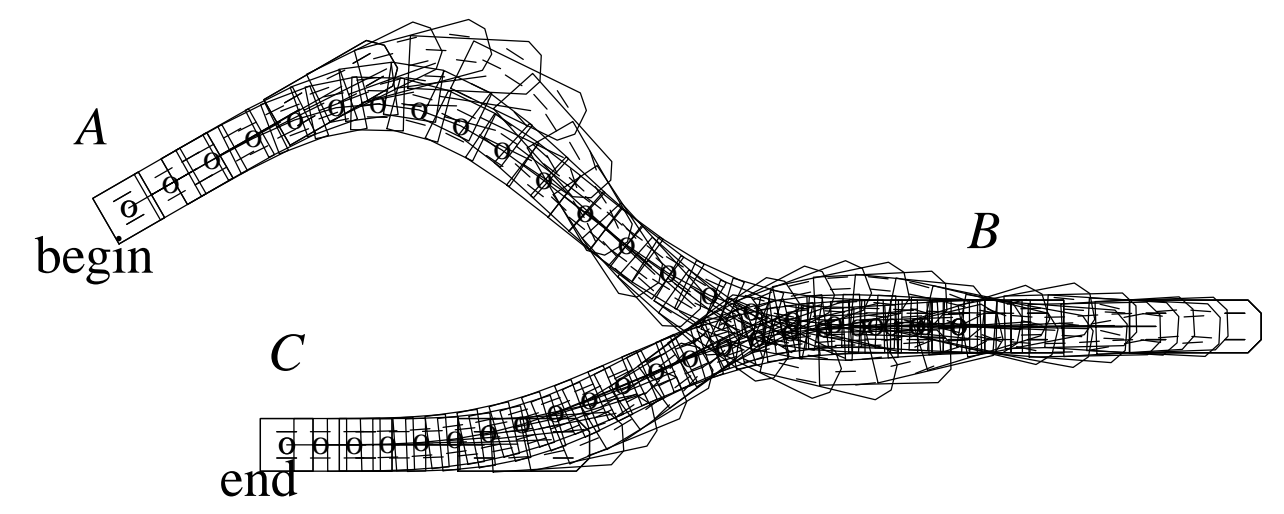

Figure 6: the successive motions of the car with two trailers.

We have

$$
\kappa_{1}=\frac{1}{\sqrt{1+d_{2}^{2} \kappa_{2}^{2}}}\left(\kappa_{2}+\frac{d_{2}}{1+d_{2}^{2} \kappa_{2}^{2}} \frac{d \kappa_{2}}{d s_{2}}\right)
$$

and $d s_{1}=\sqrt{1+d_{2}^{2} \kappa_{2}^{2}} d s_{2}$. Similarly,

$$
\kappa_{0}=\frac{1}{\sqrt{1+d_{1}^{2} \kappa_{1}^{2}}}\left(\kappa_{1}+\frac{d_{1}}{1+d_{1}^{2} \kappa_{1}^{2}} \frac{d \kappa_{1}}{d s_{1}}\right)
$$

and $d s_{0}=\sqrt{1+d_{1}^{2} \kappa_{1}^{2}} d s_{1}$. Thus $u_{1}$ is given explicitly by

$$
u_{1}=\frac{d s_{0}}{d t}=\sqrt{1+d_{1}^{2} \kappa_{1}^{2}} \sqrt{1+d_{2}^{2} \kappa_{2}^{2}} \sqrt{1+(d f / d x)^{2}} \dot{x}(t)
$$

where $[0, T] \ni t \rightarrow x(t)$ is any increasing smooth time function. $(x(0), f(x(0)))(\operatorname{resp} .(x(T), f(x(T))))$ are the coordinates of $A$ (resp. $B$ ) and $\dot{x}(0)=\dot{x}(T)=0$. Since $\tan (\phi)=d_{0} \kappa_{0}$, we get

$$
u_{2}=\frac{d \phi}{d t}=\frac{d_{0}}{1+d_{0}^{2} \kappa_{0}^{2}} \frac{d \kappa_{0}}{d s_{0}} u_{1} .
$$

Here, we are not actually concerned with obstacles. The fact that the internal configuration depends only on the curvature results from the general following property: a plane curve is entirely defined (up to rotation and translation) by its curvature. For the $n$-trailer case, the angles $\theta_{n}-\theta_{n-1}, \ldots, \theta_{1}-\theta_{0}$ and $\phi$ describing the relative configuration of the system are only functions of $\kappa_{n}$ and its first $n$-derivatives with respect to $s_{n}$. 
Consequently, limitations due to obstacles can be expressed up to a translation (defined by $P_{n}$ ) and a rotation (defined by the tangent direction $\frac{d P_{n}}{d s_{n}}$ ) via $\kappa_{n}$ and its first $n$-derivatives with respect to $s_{n}$. Such considerations can be of some help in finding a curve avoiding collisions. More details on obstacle avoidance can be found in (Laumond et al. 1993) where a car without trailer is considered.

The multi-steering trailer systems considered in (Bushnell et al. 1993), (Tilbury et al. 1993), (Tilbury and Chelouah 1993) are also flat: the flat output is then obtained by adding to the Cartesian coordinates of the last trailer, the angles of the trailers that are directly steered. This generalization is quite natural in view of the geometric construction of figure 4.

\section{High-frequency control of non-flat systems}

We address here a method for controlling non-flat systems via their approximations by averaged and flat ones. More precisely, we develop on three examples an idea due to the Russian physicist Kapitsa (Bogaevski and Povzner 1991, Landau and Lifshitz 1982, Sagdeev et al. 1988). He considers the motion of a particle in a highly oscillating field and proposes a method for deriving the equations of the averaged motion and potential. He shows that the inverted position of a single pendulum is "stabilized" when the suspension point oscillates rapidly. Notice that some related calculations may be found in (Baillieul 1993). For the use of high-frequency control in different contexts see also (Bentsman 1987, Meerkov 1980, Sussmann and Liu 1991).

(Acheson 1993, Stephenson 1908)

\subsection{The Kapitsa pendulum}

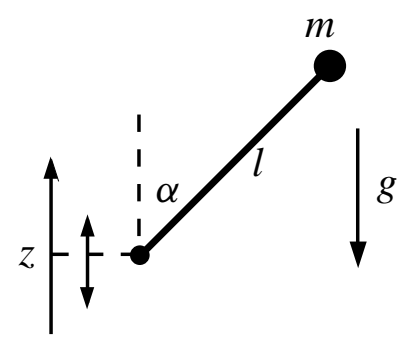

Figure 7: The Kapitsa pendulum: the suspension point oscillates rapidly on a vertical axis. 
The notation are summarized on figure 7. We assume that the vertical velocity $\dot{z}=u$ of the suspension point is the control. The equations of motion are:

$$
\left\{\begin{aligned}
\dot{\alpha} & =p+\frac{u}{l} \sin \alpha \\
\dot{p} & =\left(\frac{g}{l}-\frac{u^{2}}{l^{2}} \cos \alpha\right) \sin \alpha-\frac{u}{l} p \cos \alpha \\
\dot{z} & =u
\end{aligned}\right.
$$

where $p$ is proportional to the generalized impulsion; $g$ and $l$ are physical constants. This system is not flat since it admits only one control variable and is not linearizable via static feedback (Charlet et al. 1989). However it is strongly accessible.

We state

$$
u=u_{1}+u_{2} \cos (t / \varepsilon)
$$

where $u_{1}$ and $u_{2}$ are auxiliary control and $0<\varepsilon \ll \sqrt{l / g}$. It is then natural to consider the following averaged control system:

$$
\left\{\begin{aligned}
\dot{\bar{\alpha}} & =\bar{p}+\frac{u_{1}}{l} \sin \bar{\alpha} \\
\dot{\bar{p}} & =\left(\frac{g}{l}-\frac{\left(u_{1}\right)^{2}}{l^{2}} \cos \bar{\alpha}-\frac{\left(u_{2}\right)^{2}}{2 l^{2}} \cos \bar{\alpha}\right) \sin \bar{\alpha}-\frac{u_{1}}{l} \bar{p} \cos \bar{\alpha} \\
\dot{\bar{z}} & =u_{1} .
\end{aligned}\right.
$$

It admits two control variables, $u_{1}$ and $u_{2}$, whereas the original system (19) admits only one, $u$. Moreover $(20)$ is flat with $(\bar{\alpha}, \bar{z})$ as linearizing output.

The endogenous dynamic feedback

$$
\left\{\begin{aligned}
\dot{\xi} & =v_{1} \\
u_{1} & =\xi \\
u_{2} & =\sqrt{\frac{2 l}{\cos \bar{\alpha}}\left(g+v_{1}\right)-\frac{2 l^{2}}{\cos \bar{\alpha} \sin \bar{\alpha}} v_{2}}
\end{aligned}\right.
$$

transforms (20) into

$$
\left\{\begin{array}{c}
\ddot{\bar{z}}=v_{1} \\
\ddot{\bar{\alpha}}=v_{2} .
\end{array}\right.
$$

Set

$$
\left\{\begin{array}{l}
v_{1}=-\left(\frac{1}{\tau_{1}}+\frac{1}{\tau_{2}}\right) \xi-\frac{1}{\tau_{1} \tau_{2}}\left(\bar{z}-z^{s p}\right) \\
v_{2}=-\left(\frac{1}{\tau_{1}}+\frac{1}{\tau_{2}}\right)\left(\bar{p}+\frac{\xi}{l} \sin \bar{\alpha}\right)-\frac{1}{\tau_{1} \tau_{2}}\left(\bar{\alpha}-\alpha^{s p}\right)
\end{array}\right.
$$

where the parameters $\tau_{1}, \tau_{2}>0$ and $\left.\alpha^{s p} \in\right]-\pi / 2, \pi / 2[/\{0\}$. Then, the closed-loop averaged system $(20,21,23)$ admits an hyperbolic equilibrium point characterized by $\left(z^{s p}, \alpha^{s p}\right)$ that is asymptotically stable. 
Consider now (19) and the high-frequency control $u=u_{1}+u_{2} \sin (t / \varepsilon)$ with $0<\varepsilon \ll \sqrt{l / g}$ and $\left(u_{1}, u_{2}\right)$ given by $(21,23)$ where $\bar{\alpha}, \bar{p}$ and $\bar{z}$ are replaced by $\alpha, p$ and $z$. Then, the corresponding averaged system is nothing but (22) with $v_{1}$ and $v_{2}$ given by (23). Since the averaged system admits a hyperbolic asymptotically stable equilibrium, the perturbed system admits an hyperbolic asymptotically stable limit cycle around $(\alpha, p, z)=\left(\alpha^{s p}, 0, z^{s p}\right)$ (Guckenheimer and Holmes 1983, theorem 4.1.1, page 168): such control maintains $(z, \alpha)$ near $\left(z^{s p}, \alpha^{s p}\right)$. Moreover this control method is robust in the following sense: the existence and the stability of the limit cycle is not destroyed by small static errors in the parameters $l$ and $g$ and in the measurements of $\alpha, p, z$ and $u$.

As illustrated by the simulations of figure 8, the generalization to trajectory tracking for $\alpha$ and $z$ is straightforward. These simulations give also a rough estimate of the errors that can be tolerated. The system parameter values are $l=0.10 \mathrm{~m}$ and $g=9.81 \mathrm{~ms}^{-2}$. The design control parameters are $\varepsilon=0.025 / 2 \pi \mathrm{s}$ and $\tau_{1}=\tau_{2}=0.10 \mathrm{~s}$. For the two upper graphics of figure 8 , no error is introduced: control is computed with $l=0.10 \mathrm{~m}$ and $g=9.81 \mathrm{~ms}^{-2}$. For the two lower graphics of figure 8 , parameter errors are introduced: control is computed with with $l=0.11 \mathrm{~m}$ and $g=9.00 \mathrm{~ms}^{-2}$. 

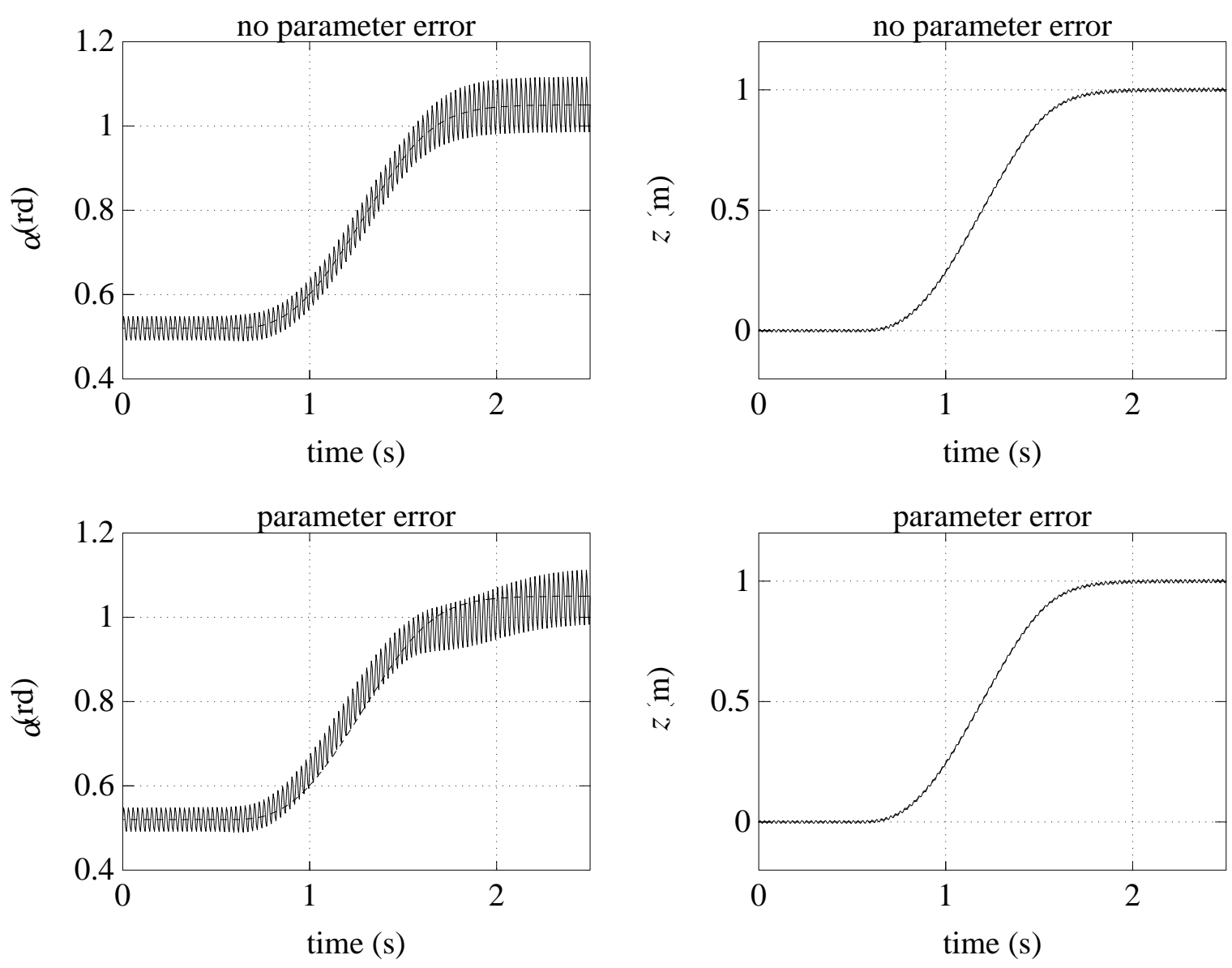

Figure 8: Robustness test of the high-frequency control for the inverted pendulum. 


\subsection{The variable-length pendulum}

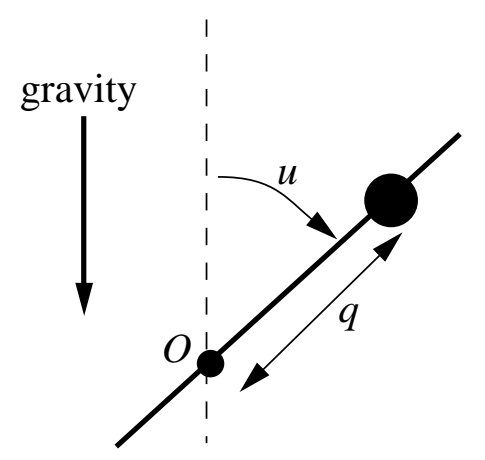

Figure 9: pendulum with variable-length.

Let us consider the variable-length pendulum of (Bressan and Rampazzo 1993). The notations are summarized on figure 9. We assume as in (Bressan and Rampazzo 1993) that the velocity $\dot{u}=v$ is the control. The equations of motion are:

$$
\left\{\begin{array}{l}
\dot{q}=p \\
\dot{p}=-\cos u+q v^{2} \\
\dot{u}=v
\end{array}\right.
$$

where mass and gravity are normalized to 1 .

This system is not flat since it admits only one control variable and is not linearizable via static feedback (Charlet et al. 1989). It is, however, strongly accessible.

As for the Kapitsa pendulum, we set

$$
v=v_{1}+v_{2} \cos (t / \varepsilon)
$$

where $v_{1}$ and $v_{2}$ are auxiliary controls, $0<\varepsilon \ll 1$. We consider the averaged control system:

$$
\left\{\begin{array}{l}
\dot{\bar{q}}=\bar{p} \\
\dot{\bar{p}}=-\cos \bar{u}+q\left(v_{1}\right)^{2}+q\left(v_{2}\right)^{2} / 2 \\
\dot{\bar{u}}=v_{1} .
\end{array}\right.
$$

This system is obviously linearizable via static feedback with $(\bar{q}, \bar{u})$ as linearizing output.

The static feedback

$$
\left\{\begin{array}{l}
v_{1}=w_{1} \\
v_{2}=\sqrt{2\left(\frac{w_{2}+\cos \bar{u}}{\bar{q}}-\left(w_{1}\right)^{2}\right)}
\end{array}\right.
$$


transforms (25) into

$$
\left\{\begin{array}{l}
\dot{\bar{u}}=w_{1} \\
\ddot{\bar{q}}=w_{2}
\end{array}\right.
$$

Set

$$
\left\{\begin{array}{l}
w_{1}=-\frac{\bar{u}-u^{s p}}{\tau_{1}} \\
w_{2}=-\left(\frac{1}{\tau_{1}}+\frac{1}{\tau_{2}}\right) \bar{p}-\frac{1}{\tau_{1} \tau_{2}}\left(\bar{q}-q^{s p}\right)
\end{array}\right.
$$

with $\left.\tau_{1}, \tau_{2}>0, u^{s p} \in\right]-\pi / 2, \pi / 2\left[, q^{s p}>0\right.$. The closed-loop averaged system $(25,26,28)$ admits an hyperbolic equilibrium point $\left(u^{s p}, q^{s p}\right)$, which is asymptotically stable.

Similarly to the Kapitsa pendulum, the control law is as follows: $v=v_{1}+v_{2} \sin (t / \varepsilon), 0<\varepsilon \ll 1$; $\left(v_{1}, v_{2}\right)$ is given by $(26,28)$ where $\bar{q}, \bar{p}$ and $\bar{u}$ are replaced by $q, p$ and $u$. This control strategy leads to a small and attractive limit cycle. As illustrated by the simulations of figure 10, the size of these limit cycle is an increasing function of $\varepsilon$ and tends to 0 as $\varepsilon$ tends to $0^{+}$. The design control parameters are $\tau_{1}=0.5, \tau_{2}=0.4$. 

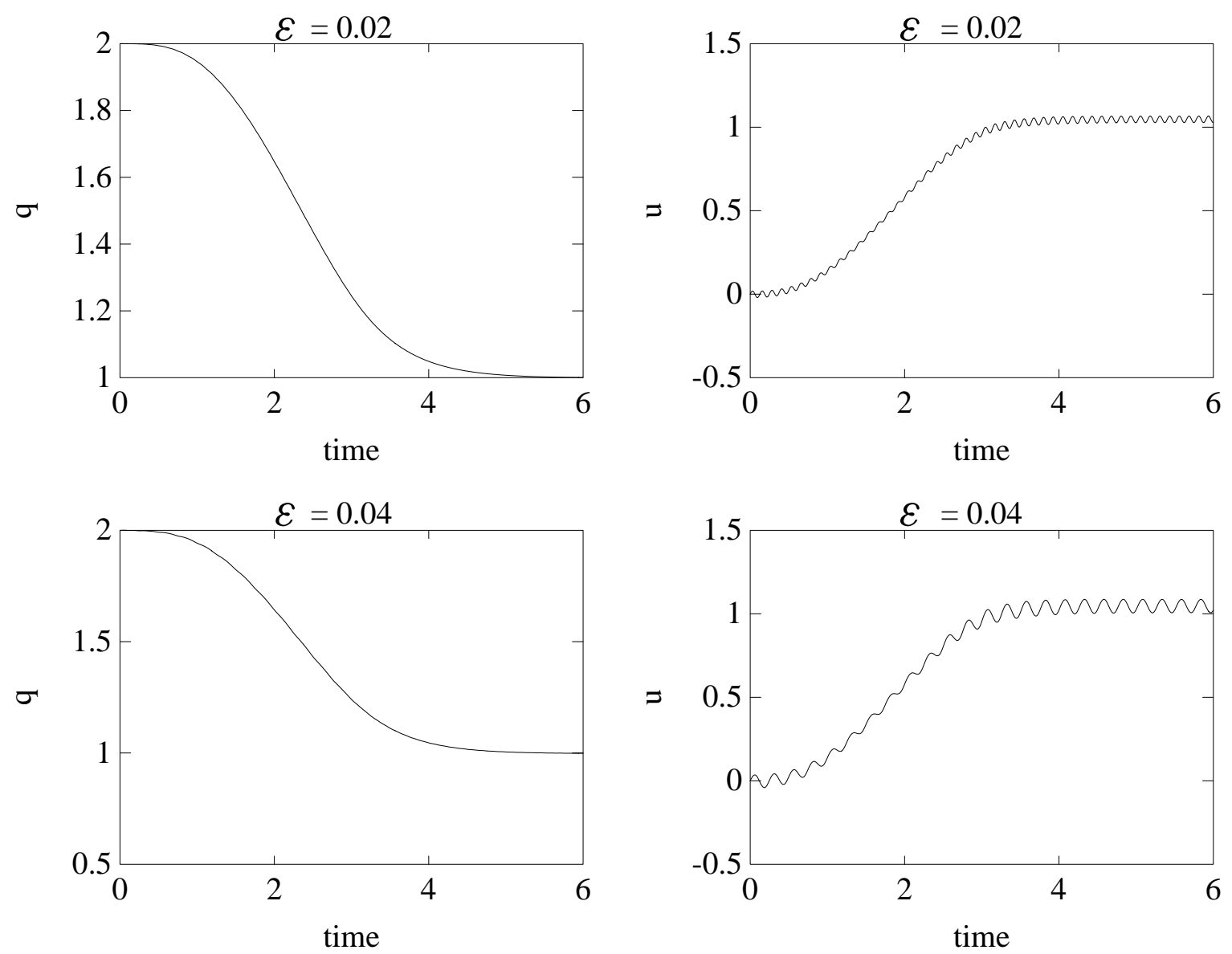

Figure 10: high-frequency control for the variable-length pendulum. 


\subsection{The inverted double pendulum}

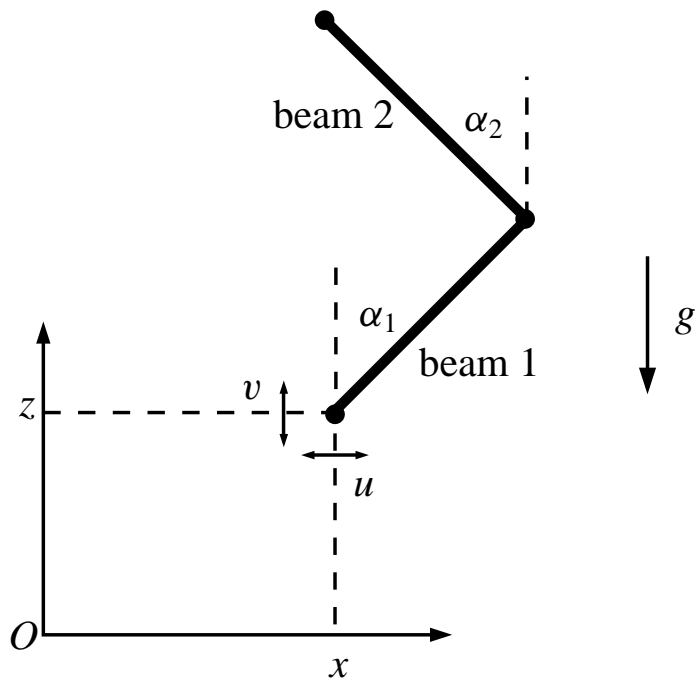

Figure 11: The inverted double pendulum: the horizontal velocity $u$ and vertical velocity $v$ of the suspension point are the two control variables.

The double inverted pendulum of figure 11 moves in a vertical plane. Assume that $u$ (resp. $v$ ) the horizontal (resp. vertical) velocity of the suspension point $(x, z)$ is a control variable. The equations of motion are (implicit form):

$$
\left\{\begin{aligned}
p_{1} & =I_{1} \dot{\alpha}_{1}+I \dot{\alpha}_{2} \cos \left(\alpha_{1}-\alpha_{2}\right)+n_{1} \dot{x} \cos \alpha_{1}-n_{1} \dot{z} \sin \alpha_{1} \\
p_{2} & =I \dot{\alpha}_{1} \cos \left(\alpha_{1}-\alpha_{2}\right)+I_{2} \dot{\alpha}_{2}+n_{2} \dot{x} \cos \alpha_{2}-n_{2} \dot{z} \sin \alpha_{2} \\
\dot{p}_{1} & =n_{1} g \sin \alpha_{1}-n_{1} \dot{\alpha}_{1} \dot{x} \sin \alpha_{1}-n_{1} \dot{\alpha}_{1} \dot{z} \cos \alpha_{1} \\
\dot{p}_{2} & =n_{2} g \sin \alpha_{2}-n_{2} \dot{\alpha}_{2} \dot{x} \sin \alpha_{2}-n_{2} \dot{\alpha}_{2} \dot{z} \cos \alpha_{2} \\
\dot{x} & =u \\
\dot{z} & =v
\end{aligned}\right.
$$

where $p_{1}$ and $p_{2}$ are the generalized impulsions associated to the generalized coordinates $\alpha_{1}$ and $\alpha_{2}$, respectively. The quantities $g, I, I_{1}, I_{2}, n_{1}$ and $n_{2}$ are constant physical parameters:

$$
I_{1}=\left(\frac{m_{1}}{3}+m_{2}\right)\left(l_{1}\right)^{2}, \quad I_{2}=\frac{m_{2}}{3}\left(l_{2}\right)^{2}, \quad I=\frac{m_{2}}{2} l_{1} l_{2}, \quad n_{1}=\left(\frac{m_{1}}{2}+m_{2}\right) l_{1}, \quad n_{2}=\frac{m_{2}}{2} l_{2},
$$

where $m_{1}$ and $m_{2}$ (resp. $l_{1}$ and $l_{2}$ ) are the masses (resp. lengths) of beams 1 and 2 which are assumed to be homogeneous.

Proposition 6 System (29) with the two control variables $u$ and $v$, is not flat. 
Proof The proof is just an application of the necessary flatness condition of theorem 3. Since $u=\dot{x}$ and $v=\dot{z},(29)$ is flat if, and only if, the reduced system,

$$
\left\{\begin{array}{l}
p_{1}=I_{1} \dot{\alpha}_{1}+I \dot{\alpha}_{2} \cos \left(\alpha_{1}-\alpha_{2}\right)+n_{1} \dot{x} \cos \alpha_{1}-n_{1} \dot{z} \sin \alpha_{1} \\
p_{2}=I \dot{\alpha}_{1} \cos \left(\alpha_{1}-\alpha_{2}\right)+I_{2} \dot{\alpha}_{2}+n_{2} \dot{x} \cos \alpha_{2}-n_{2} \dot{z} \sin \alpha_{2} \\
\dot{p}_{1}=n_{1} g \sin \alpha_{1}-n_{1} \dot{\alpha}_{1} \dot{x} \sin \alpha_{1}-n_{1} \dot{\alpha}_{1} \dot{z} \cos \alpha_{1} \\
\dot{p}_{2}=n_{2} g \sin \alpha_{2}-n_{2} \dot{\alpha}_{2} \dot{x} \sin \alpha_{2}-n_{2} \dot{\alpha}_{2} \dot{z} \cos \alpha_{2}
\end{array}\right.
$$

is flat. Denote symbolically by $F(\xi, \dot{\xi})=0$ the equations (30) where $\xi=\left(\alpha_{1}, \alpha_{2}, x, z, p_{1}, p_{2}\right)$. Consider $(\xi, \zeta)$ such that $F(\xi, \zeta)=0$. We are looking for a vector $a=\left(a_{\alpha_{1}}, a_{\alpha_{2}}, a_{x}, a_{z}, a_{p_{1}}, a_{p_{2}}\right)$ such that, for all $\lambda \in \mathbb{R}, F(\xi, \zeta+\lambda a)=0$. The second order conditions, $\left.\frac{d^{2}}{d \lambda^{2}}\right|_{\lambda=0} F(\xi, \zeta+\lambda a)=0$, lead to

$$
a_{\alpha_{1}}\left(a_{x} \sin \alpha_{1}+a_{z} \cos \alpha_{1}\right)=0, \quad a_{\alpha_{2}}\left(a_{x} \sin \alpha_{2}+a_{z} \cos \alpha_{2}\right)=0 .
$$

Two first order conditions, $\left.\frac{d}{d \lambda}\right|_{\lambda=0} F(\xi, \zeta+\lambda a)=0$, are

$$
\left\{\begin{array}{l}
-a_{x} \cos \alpha_{1}+a_{z} \sin \alpha_{1}=\frac{I_{1}}{n_{1}} a_{\alpha_{1}}+\frac{I}{n_{1}} \cos \left(\alpha_{1}-\alpha_{2}\right) a_{\alpha_{2}} \\
-a_{x} \cos \alpha_{2}+a_{z} \sin \alpha_{2}=\frac{I}{n_{2}} \cos \left(\alpha_{1}-\alpha_{2}\right) a_{\alpha_{1}}+\frac{I_{2}}{n_{2}} a_{\alpha_{2}}
\end{array}\right.
$$

Simple computations show that, if $\frac{I}{n_{1}} \neq \frac{I_{2}}{n_{2}}$ and $\frac{I_{1}}{n_{1}} \neq \frac{I}{n_{2}}$ (these conditions are always satisfied for homogeneous identical beams), then $\left(a_{\alpha_{1}}, a_{\alpha_{2}}, a_{x}, a_{z}\right)=0$. The two remaining first order conditions imply that $\left(a_{p_{1}}, a_{p_{2}}\right)=0$. Thus $a=0$ and the inverted double pendulum is not flat.

The same control method as the one explained in details for the Kapitsa pendulum (19) can be also used for the double pendulum. The only difference relies on the calculations that are here more tedious. We just sketch some simulations (Fliess et al. 1993b).

To approximate the non-flat system (29) by a flat one, we set $u=u_{1}+u_{2} \cos (t / \varepsilon)$ and $v=$ $v_{1}+v_{2} \cos (t / \varepsilon)$ where $0<\varepsilon \ll \min \left(\sqrt{\frac{I_{1}}{n_{1} g}}, \sqrt{\frac{I_{2}}{n_{2} g}}\right)$ and $u_{1}, u_{2}, v_{1}, v_{2}$ are new control variables. This leads to a flat averaged system with $\left(\alpha_{1}, \alpha_{2}, x, z\right)$ as the linearizing output. The endogenous dynamic feedback that linearized the averaged system provides then $\left(u_{1}, u_{2}, v_{1}, v_{2}\right)$. For the simulations of figure 12, the angles $\alpha_{1}$ and $\alpha_{2}$ follow approximately prescribed trajectories whereas, simultaneously, the suspension point $(x, z)$ is maintained approximately constant. 

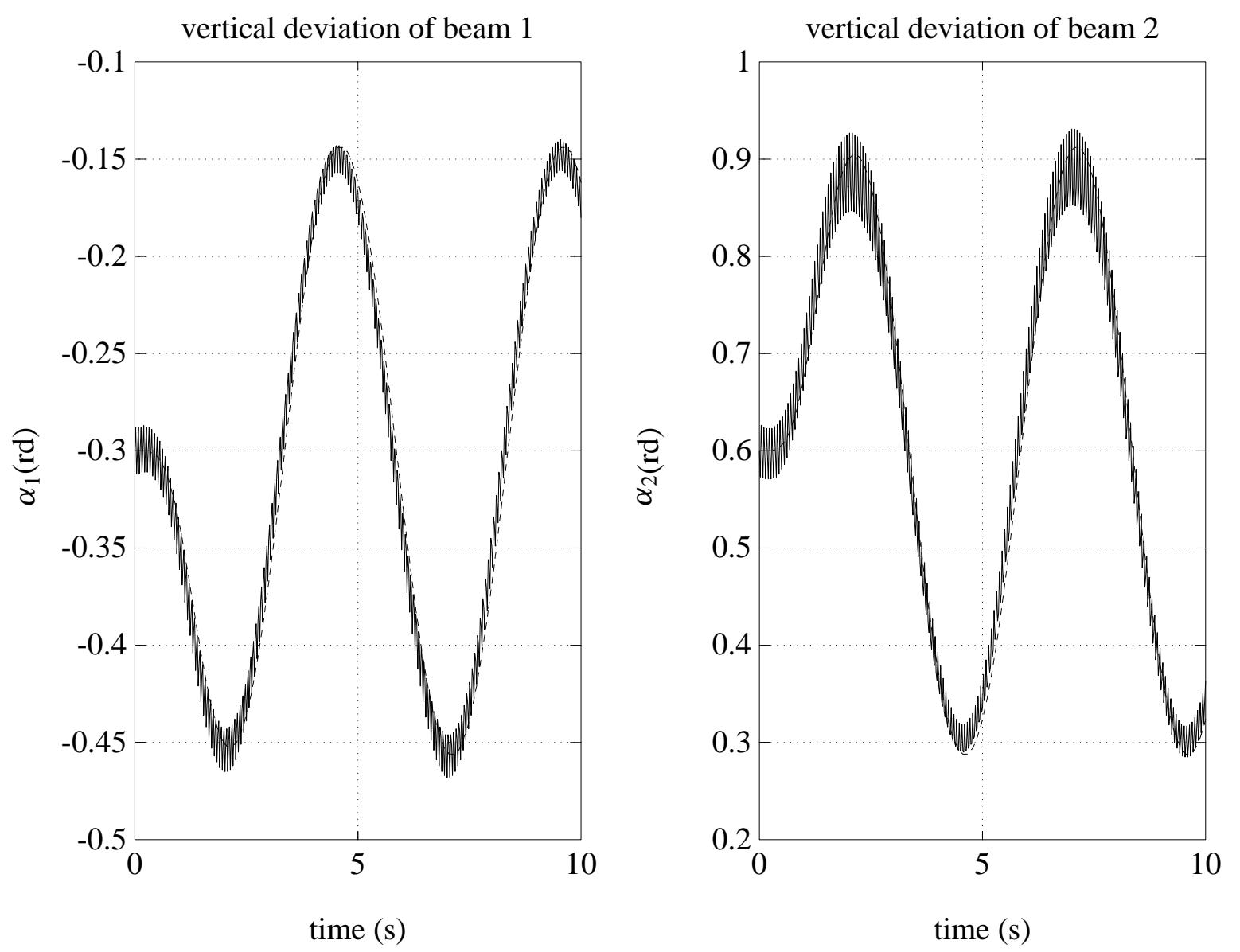

Figure 12: Simulation of the inverted double pendulum via high-frequency control. 


\section{Conclusion}

Our five examples, as well as other ones in preparation in various domains of engineering, indicate that flatness and defect ought to be considered as physical and/or geometric properties. This explains why flat systems are so often encountered in spite of the non-genericity of dynamic feedback linearizability in some customary mathematical topologies (Tchoń 1994, Rouchon 1994).

We hope to have convinced the reader that flatness and defect bring a new theoretical and practical insight in control. We briefly list some important open problems:

- Ritt's work (Ritt 1950) shows that differential algebra provides powerful algorithmic means (see (Diop 1991, Diop 1992) for a survey and connections with control). Can flatness and defect be determined by this kind of procedures?

- great progress have recently been made in nonlinear time-varying feedback stabilization (see, e.g., (Coron 1992, Coron 1994)). Most of the examples which were considered happen to be flat (see, e.g., (Coron and D'Andréa-Novel 1992)). The utilization of this property is related to the understanding of the notion of singularity (see, e.g., (Martin 1993) for a first step in this direction and the references therein).

- the two averaged systems associated to high-frequency control are flat. Can this result be generalized to a large class of devices?

- differential algebra is not the only possible language for investigating flatness and defect. The extension of the differential algebraic formalism to smooth and analytic functions (Jakubczyk 1992) and the differential geometric approach (Martin 1992, Fliess et al. 1993d, Fliess et al. 1993e, Pomet 1993) should also be examined in this context.

\section{References}

Acheson, D. 1993. A pendulum theorem. Proc. R. Soc. Lond. A 443, 239-245.

Baillieul, J. 1993. Stable average motions of mechanical systems subject to periodic forcing. preprint.

Bentsman, J. 1987. Vibrational control of a class of nonlinear multiplicative vibrations. IEEE Trans. Automat. Control 32, 711-716.

Bogaevski, V. and A. Povzner 1991. Algebraic Methods in Nonlinear Perturbation Theory. Springer, New York.

Bressan, A. and F. Rampazzo 1993. On differential systems with quadratic impulses and their applications to Lagrangian mechanics. SIAM J. Control Optimization 31, 1205-1220.

Bushnell, L., D. Tilbury and S. Sastry 1993. Steering chained form nonholonomic systems using sinusoids: the firetruck example.. In Proc. ECC'93, Groningen. pp. 1432-1437. 
Campion, G., B. D’Andréa-Novel, G. Bastin and C. Samson 1992. Modeling and feedback control of wheeled mobile robots. In Lecture Note of the Summer School on Theory of Robots, Grenoble.

Cartan, E. 1915. Sur l'intégration de certains systèmes indéterminés d'équations différentielles. $J$. für reine und angew. Math. 145, 86-91. also in Oeuvres Complètes, part II, vol 2, pp.1164-1174, CNRS, Paris, 1984.

Charlet, B., J. Lévine and R. Marino 1989. On dynamic feedback linearization. Systems Control Letters 13, 143-151.

Charlet, B., J. Lévine and R. Marino 1991. Sufficient conditions for dynamic state feedback linearization. SIAM J. Control Optimization 29, 38-57.

Claude, D. 1986. Everything you always wanted to know about linearization. In M. Fliess and M. Hazewinkel (Eds.). Algebraic and Geometric Methods in Nonlinear Control Theory. Reidel, Dordrecht. pp. 181-226.

Cohn, P. 1985. Free Rings and their Relations. second edn. Academic Press, London.

Coron, J. 1992. Global stabilization for controllable systems without drift. Math. Control Signals Systems 5, 295-312.

Coron, J. 1994. Linearized control systems and applications to smooth stabilization. SIAM J. Control Optimization.

Coron, J. and B. D'Andréa-Novel 1992. Smooth stabilizing time-varying control laws for a class of nonlinear systems: application to mobile robots. In Proc. IFAC-Symposium NOLCOS'92, Bordeaux. pp. 658-672.

D’Andréa-Novel, B. and J. Lévine 1990. Modelling and nonlinear control of an overhead crane. In M. Kashoek, J. van Schuppen and A. Rand (Eds.). Robust Control of Linear and Nonlinear Systems, MTNS'89. Vol. II. Birkhäuser, Boston. pp. 523-529.

D’Andréa-Novel, B., G. Bastin and G. Campion 1992a. Dynamic feedback linearization of nonholonomic wheeled mobile robots. In IEEE Conference on Robotics and Automation, Nice. pp. 25272532.

D’Andréa-Novel, B., Ph. Martin and R. Sépulchre 1992b. Full dynamic feedback linearization of a class of mechanical systems. In H. Kimura and S. Kodama (Eds.). Recent Advances in Mathematical Theory of Systems, Control, Network and Signal Processing II (MTNS-91, Kobe, Japan), Mita Press, Tokyo. pp. 327-333.

Delaleau, E. and M. Fliess 1992. Algorithme de structure, filtrations et découplage. C.R. Acad. Sci. Paris I-315, 101-106. 
Di Benedetto, M., J.W. Grizzle and C.H. Moog 1989. Rank invariants of nonlinear systems. SIAM J. Control Optimization 27, 658-672.

Diop, S. 1991. Elimination in control theory. Math. Control Signals Systems 4, 17-32.

Diop, S. 1992. Differential-algebraic decision methods and some applications to system theory. Theoret. Comput. Sci. 98, 137-161.

Dubrovin, B., A.T. Fomenko and S.P. Novikov 1984. Modern Geometry-Methods and Applications - Part I. Springer, New York.

Fliess, M. 1989. Automatique et corps différentiels. Forum Math. 1, 227-238.

Fliess, M. 1990a. Generalized controller canonical forms for linear and nonlinear dynamics. IEEE Trans. Automat. Control 35, 994-1001.

Fliess, M. 1990b. Some basic structural properties of generalized linear systems. Systems Control Letters 15, 391-396.

Fliess, M. 1992. A remark on Willem's trajectory characterization of linear controllability. Systems Control Letters 19, 43-45.

Fliess, M. and M. Hasler 1990. Questioning the classical state space description via circuit examples. In M. Kashoek, J. van Schuppen and A. Ran (Eds.). Realization and Modelling in System Theory, MTNS'89. Vol. I. Birkhäuser, Boston. pp. 1-12.

Fliess, M. and S.T. Glad 1993. An algebraic approach to linear and nonlinear control. In H. Trentelman and J. Willems (Eds.). Essays on Control: Perspectives in the Theory and its Applications. Birkhäuser, Boston. pp. 223-267.

Fliess, M., J. Lévine and P. Rouchon 1991. A simplified approach of crane control via a generalized state-space model. In Proc. 30th IEEE Control Decision Conf., Brighton. pp. 736-741.

Fliess, M., J. Lévine and P. Rouchon 1993a. A generalized state variable representation for a simplified crane description. Int. J. Control 58, 277-283.

Fliess, M., J. Lévine, Ph. Martin and P. Rouchon 1992a. On differentially flat nonlinear systems. In Proc. IFAC-Symposium NOLCOS'92, Bordeaux. pp. 408-412.

Fliess, M., J. Lévine, Ph. Martin and P. Rouchon 1992b. Sur les systèmes non linéaires différentiellement plats. C.R. Acad. Sci. Paris I-315, 619-624.

Fliess, M., J. Lévine, Ph. Martin and P. Rouchon 1993b. Défaut d'un système non linéaire et commande haute fréquence. C.R. Acad. Sci. Paris I-316, 513-518. 
Fliess, M., J. Lévine, Ph. Martin and P. Rouchon 1993c. Differential flatness and defect: an overview. In Workshop on Geometry in Nonlinear Control, Banach Center Publications, Warsaw.

Fliess, M., J. Lévine, Ph. Martin and P. Rouchon 1993d. Linéarisation par bouclage dynamique et transformations de Lie-Bäcklund. C.R. Acad. Sci. Paris I-317, 981-986.

Fliess, M., J. Lévine, Ph. Martin and P. Rouchon 1993e. Towards a new differential geometric setting in nonlinear control. In Proc. Internat. Geometric. Coll., Moscow.

Guckenheimer, J. and P. Holmes 1983. Nonlinear Oscillations, Dynamical Systems and Bifurcations of Vector Fields. Springer, New York.

Hartshorne, R. 1977. Algebraic Geometry. Springer, New York.

Hilbert, D. 1912. Über den Begriff der Klasse von Differentialgleichungen. Math. Ann. 73, 95-108. also in Gesammelte Abhandlungen, vol. III, pp. 81-93, Chelsea, New York, 1965.

Isidori, A. 1989. Nonlinear Control Systems. 2nd edn. Springer, New York.

Jacobson, N. 1985. Basic Algebra, I and II. 2nd edn. Freeman, New York.

Jakubczyk, B. 1992. Remarks on equivalence and linearization of nonlinear systems. In Proc. IFACSymposium NOLCOS'92, Bordeaux. pp. 393-397.

Johnson, J. 1969. Kähler differentials and differential algebra. Ann. Math. 89, 92-98.

Kailath, T. 1980. Linear Systems. Prentice-Hall, Englewood Cliffs, NJ.

Kolchin, E. 1973. Differential Algebra and Algebraic Groups. Academic Press, New York.

Landau, L. and E. Lifshitz 1982. Mechanics. 4th edn. Mir, Moscow.

Laumond, J. 1991. Controllability of a multibody mobile robot. In IEEE International Conf. on advanced robotics, 91 ICAR. pp. 1033-1038.

Laumond, J., P.E. Jacobs, M. Taix and R.M. Murray 1993. A motion planner for nonholonomic mobile robots. preprint.

Li, C.-W. and Y.-K. Feng 1987. Functional reproducibility of general multivariable analytic nonlinear systems. Int. J. Control 45, 255-268.

Martin, P. 1992. Contribution à l'étude des systèmes diffèrentiellement plats. PhD thesis. École des Mines de Paris.

Martin, P. 1993. An intrinsic condition for regular decoupling. Systems Control Letters 20, 383-391. 
Martin, P. and P. Rouchon 1993. Systems without drift and flatness. In Proc. MTNS 93, Regensburg, Germany.

Marttinen, A., J. Virkkunen and R.T. Salminen 1990. Control study with a pilot crane. IEEE Trans. Edu. 33, 298-305.

Meerkov, S. 1980. Principle of vibrational control: theory and applications. IEEE Trans. Automat. Control 25, 755-762.

Monaco, S. and D. Normand-Cyrot 1992. An introduction to motion planning under multirate digital control. In Proc. 31th IEEE Control Decision Conf.,Tucson. pp. 1780-1785.

Moog, C., J. Perraud, P. Bentz and Q.T. Vo 1989. Prime differential ideals in nonlinear rational control systems. In Proc. NOLCOS'89, Capri, Italy. pp. 178-182.

Murray, R. and S.S. Sastry 1993. Nonholonomic motion planning: Steering using sinusoids. IEEE Trans. Automat. Control 38, 700-716.

Nijmeijer, H. and A.J. van der Schaft 1990. Nonlinear Dynamical Control Systems. Springer, New York.

Pomet, J. 1993. A differential geometric setting for dynamic equivalence and dynamic linearization. In Workshop on Geometry in Nonlinear Control, Banach Center Publications, Warsaw.

Ritt, J. 1950. Differential Algebra. Amer. Math. Soc., New York.

Rouchon, P. 1994. Necessary condition and genericity of dynamic feedback linearization. J. Math. Systems Estim. Control. In press.

Rouchon, P., M. Fliess, J. Lévine and Ph. Martin 1993a. Flatness and motion planning: the car with n-trailers.. In Proc. ECC'93, Groningen. pp. 1518-1522.

Rouchon, P., M. Fliess, J. Lévine and Ph. Martin 1993b. Flatness, motion planning and trailer systems. In Proc. 32nd IEEE Conf. Decision and Control, San Antonio. pp. 2700-2705.

Sagdeev, R., D.A. Usikov and G.M. Zaslavsky 1988. Nonlinear Physics. Harwood, Chur.

Seidenberg, A. 1952. Some basic theorems in differential algebra (characteristic p, arbitrary). Trans. Amer. Math. Soc. 73, 174-190.

Shadwick, W. 1990. Absolute equivalence and dynamic feedback linearization. Systems Control Letters 15, 35-39.

Sontag, E. 1988. Finite dimensional open loop control generator for nonlinear control systems. Internat. J. Control 47, 537-556. 
Sontag, E. 1992. Universal nonsingular controls. Systems Controls Letters 19, 221-224.

Stephenson, A. 1908. On induced stability. Phil. Mag. 15, 233-236.

Sussmann, H. and V. Jurdjevic 1972. Controllability of nonlinear systems. J. Differential Equations 12, 95-116.

Sussmann, H. and W. Liu 1991. Limits of highly oscillatory controls and the approximation of general paths by admissible trajectories. In Proc. 30th IEEE Control Decision Conf., Brighton. pp. 437-442.

Tchoń, K. 1994. Towards robustness and genericity of dynamic feedback linearization. J. Math. Systems Estim. Control.

Tikhonov, A., A. Vasil'eva and A. Sveshnikov 1980. Differential Equations. Springer, New York.

Tilbury, D. and A. Chelouah 1993. Steering a three-input nonholonomic using multirate controls. In Proc. ECC'93, Groningen. pp. 1428-1431.

Tilbury, D., O. Sørdalen, L. Bushnell and S. Sastry 1993. A multi-steering trailer system: conversion into chained form using dynamic feedback. Technical Report UCB/ERL M93/55. Electronics Research Laboratory, University of California at Berkeley.

Whittaker, E. 1937. A Treatise on the Analytical Dynamics of Particules and Rigid Bodies (4th edition). Cambridge University Press, Cambridge.

Willems, J. 1991. Paradigms and puzzles in the theory of dynamical systems. IEEE Trans. Automat. Control 36, 259-294.

Winter, D. 1974. The Structure of Fields. Springer, New York.

\section{A Prime differential ideals}

We know from (Diop 1992, lemma 5.2, page 158) (see also (Moog et al. 1989)) that, for $x=$ $\left(x_{1}, \ldots, x_{n}\right)(n \geq 0)$ and $u=\left(u_{1}, \ldots, u_{m}\right)(m \geq 0)$, the differential ideal corresponding to

$$
\dot{x}_{i}=\frac{a_{i}\left(x, u, \dot{u}, \ldots, u^{\left(\alpha_{i}\right)}\right)}{b_{i}\left(x, u, \dot{u}, \ldots, u^{\left(\beta_{i}\right)}\right)}, \quad i=1, \ldots, n,
$$

where the $a_{i}$ 's and $b_{i}$ 's are polynomials over $k$, is prime. It is then immediate that the differential ideal corresponding to the tutorial example (7) is prime: set $x=\left(x_{1}, x_{2}\right)$ and $u=\left(x_{3}, x_{4}\right)$. Let us now list our five case-studies. 
Kapitsa pendulum (19) Let us replace $\alpha$ by $\sigma=\tan (\alpha / 2)$. Then, using

$$
\dot{\sigma}=\frac{1+\sigma^{2}}{2} \dot{\alpha}, \quad \cos \alpha=\frac{1-\sigma^{2}}{1+\sigma^{2}}, \quad \sin \alpha=\frac{2 \sigma}{1+\sigma^{2}},
$$

the equations (19) become explicit and rational

$$
\left\{\begin{aligned}
\dot{\sigma} & =\frac{1+\sigma^{2}}{2}\left(p+\frac{2 \sigma u}{l\left(1+\sigma^{2}\right)}\right) \\
\dot{p} & =\left(\frac{g}{l}-\frac{u^{2}\left(1-\sigma^{2}\right)}{l^{2}\left(1+\sigma^{2}\right)}\right) \frac{2 \sigma}{1+\sigma^{2}}-\frac{p u\left(1-\sigma^{2}\right)}{l\left(1+\sigma^{2}\right)} \\
\dot{z} & =u .
\end{aligned}\right.
$$

The associated differential ideal is thus prime and leads to a finitely generated differential field extension over $\mathbb{R}$.

Variable-length pendulum (24) Similar computations with $\sigma=\tan (u / 2)$ prove that the associated differential ideal is prime.

Double pendulum (29) Similar computations with $\sigma_{1}=\tan \left(\alpha_{1} / 2\right)$ and $\sigma_{2}=\tan \left(\alpha_{2} / 2\right)$ prove that the associated differential ideal is prime.

Car with $n$-trailers (18) Similar computations with $\sigma=\tan (\varphi / 2)$ and $\sigma_{i}=\tan \left(\theta_{i} / 2\right)$ prove that the associated differential ideal is prime.

Crane (17) Analogous calculations on the generalized state variable equation $R \ddot{\theta}=-2 \dot{R} \dot{\theta}-$ $\ddot{D} \cos \theta-g \sin \theta$ given in (Fliess et al. 1991, Fliess et al. 1993a) lead to a prime differential ideal.

Another more direct way for obtaining the differential field corresponding to the crane is the following. Take (17) and consider the differential field $\mathbb{R}<x, z\rangle$ generated by the two differential indeterminates $x$ and $z$. The variable $D$ belongs to $\mathbb{R}\langle x, z\rangle$ and the variable $R$ belongs to an obvious algebraic extension $\mathcal{D}$ of $\mathbb{R}\langle x, z\rangle$, which defines the system.

\section{B Dynamic feedbacks versus endogenous feedbacks}

A dynamic feedback between two systems $\mathcal{D} / k$ and $\tilde{\mathcal{D}} / k$ consists in a finitely differential extension $\mathcal{E} / k$ such that $\mathcal{D} \subset \&$ and $\tilde{\mathcal{D}} \subset \mathcal{E}$. Assume moreover that the extension $\& / \tilde{\mathcal{D}}$ is differentially algebraic. According to theorem 1 , the (non-differential) transcendence degree of $\& / \tilde{\mathcal{D}}$ is finite, say $v$. Choose a transcendence basis $z=\left(z_{1}, \ldots, z_{v}\right)$ of $8 / \tilde{\mathcal{D}}$. It yields like $(8)$ :

$$
\begin{aligned}
A_{\alpha}\left(\dot{z}_{\alpha}, z\right) & =0 \quad \alpha=1, \ldots, v \\
B(\xi, z) & =0
\end{aligned}
$$


where $\xi$ is any element of $\mathcal{E}$ and the $A_{\alpha}$ 's and $B$ are polynomials over $\tilde{\mathcal{D}}$.

The above formulas are the counterpart in the field theoretic language of the usual ones for defining general dynamic feedbacks (see, e.g., (Isidori 1989, Nijmeijer and van der Schaft 1990)). The dynamic feedback is said to be regular if, and only if, $8 / \mathcal{D}$ and $8 / \tilde{\mathcal{D}}$ are both differentially algebraic. The following generalization of proposition 2 is immediate: the systems $\mathcal{D} / k$ and $\tilde{\mathcal{D}} / k$ possess the same differential order, i.e., the same number of independent input channels.

The situation of endogenous feedbacks is recovered when $\& / \mathcal{D}$ and $\& / \mathcal{D}$ are both algebraic, i.e., $v=0$.

\section{Proof of proposition 5}

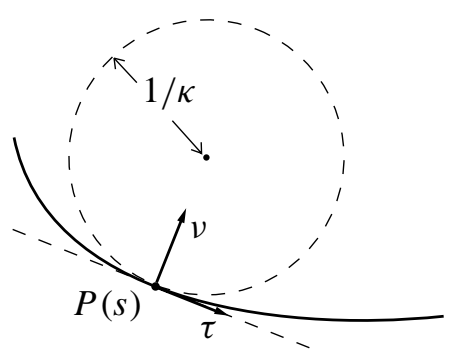

Figure 13: Frénet frame $(\tau, v)$ and curvature $\kappa$ of a smooth planar curve.

The Frénet formula Let us recall some terminology and relations relative to planar smooth curves that are displayed on figure 13 (see, e.g., (Dubrovin et al. 1984)). A curve parameterization $\mathbb{R} \ni s \rightarrow$ $P(s) \in \mathbb{R}^{2}$ is called regular if, and only if, for all $s, \frac{d P}{d s} \neq 0$. A curve is called smooth if, and only if, it admits a regular parameterization. A parameterization is called natural if, and only if, for all $s$, $\left\|\frac{d P}{d s}\right\|=1$ where $\|$.$\| denotes the Euclidian norm. For smooth curves with a natural parameterization$ $s \rightarrow P(s)$, its signed curvature $\kappa$ is defined by $\frac{d \tau}{d s}=\kappa \nu$, where $\tau=\frac{d P}{d s}$ is the unitary tangent vector and $v$ is the oriented normal vector $((\tau, v)$ is a direct orthonormal frame of the oriented Euclidian plane $\mathbb{R}^{2}$ ). Notice that $\frac{d v}{d s}=-\kappa \tau$. Every smooth curve admits a natural parameterization: every regular parameterization $t \rightarrow P(t)$ leads to a natural parameterization $s \rightarrow P(s)$ via the differential relation $d s=\left\|\frac{d P}{d t}\right\| d t$

Lemma Consider a trajectory of (18) such that the curve $\mathfrak{C}_{n}$ followed by $P_{n}$ is smooth with the natural parameterization $\left[0, L_{n}\right] \ni s_{n} \rightarrow P_{n}\left(s_{n}\right): s_{n}=0$ (resp. $s_{n}=L_{n}$ ) corresponds to the starting point (resp. end point); $L_{n}$ is the length of $\mathfrak{C}_{n}$. Assume also that for $s_{n}=0, \theta_{i-1}-\theta_{i}(i=1, \ldots, n)$ and $\phi$ belong to $]-\pi / 2, \pi / 2[$. Then, 
(i) for all $s_{n} \in\left[0, L_{n}\right], \theta_{i-1}-\theta_{i}(i=1, \ldots, n)$ and $\phi$ belong to $]-\pi / 2, \pi / 2[$.

(ii) the curves $\mathfrak{C}_{i}$ and $\mathfrak{C}$ followed by $P_{i}$ and $Q$ are $\operatorname{smooth}(i=0,1, \ldots, n)$.

(iii) $\tan \left(\theta_{i-1}-\theta_{i}\right)=d_{i} \kappa_{i}(i=1, \ldots, n)$ and $\tan \phi=d_{0} \kappa_{0}$, where $\kappa_{i}$ and $\kappa_{0}$ are the curvatures of $\mathfrak{C}_{i}$ and $\mathfrak{C}_{0}$, respectively;

(iv) the curvature $\kappa_{i}$ can be expressed as a smooth function of $\kappa_{n}$ and of its first $n-i$ derivatives with respect to $s_{n}$; moreover the mapping (which is independent of $s_{n}$ )

$$
\left(\begin{array}{c}
\kappa_{n} \\
\frac{d \kappa_{n}}{d s_{n}} \\
\vdots \\
\frac{d^{n} \kappa_{n}}{d s_{n}^{n}}
\end{array}\right) \longrightarrow\left(\begin{array}{c}
\kappa_{n} \\
\kappa_{n-1} \\
\vdots \\
\kappa_{0}
\end{array}\right)
$$

is a global diffeomorphism from $\mathbb{R}^{n+1}$ to $\mathbb{R}^{n+1}$.

Proof of the lemma As displayed on figure 4, the point $P_{i-1}$ belongs to the tangent to $\mathcal{C}_{i}$ at $P_{i}$ and at the fixed distance $d_{i}$ from $P_{i}$. By assumption $\tau_{n}=\frac{d P_{n}}{d s_{n}}$ admits the good orientation: $P_{n-1}=P_{n}+d_{n} \tau_{n}$ (we do not have $P_{n-1}=P_{n}-d_{n} \tau_{n}$ ). Thus $\mathcal{C}_{n-1}$ is given by the parameterization $s_{n} \rightarrow P_{n}+d_{n} \tau_{n}$ which is regular since $\left\|\frac{d P_{n-1}}{d s_{n}}\right\|=\sqrt{1+d_{n}^{2} \kappa_{n}^{2}}$. A natural parameterization $s_{n-1} \rightarrow P_{n-1}$ is given by

$$
d s_{n-1}=\sqrt{1+d_{n}^{2} \kappa_{n}^{2}} d s_{n}
$$

The unitary tangent vector, $\tau_{n-1}$, is given by

$$
\sqrt{1+d_{n}^{2} \kappa_{n}^{2}} \tau_{n-1}=\tau_{n}+d_{n} \kappa_{n} v_{n}
$$

where $v_{n}$ is the oriented normal to $\mathcal{C}_{n}$. The angle $\theta_{n-1}-\theta_{n}$ is the angle between $\tau_{n}$ and $\tau_{n-1}$. Thus $\tan \left(\theta_{n-1}-\theta_{n}\right)=d_{n} \kappa_{n}$. Since $\kappa_{n}$ is always finite and $\theta_{n-1}-\theta_{n}$ belongs ] $-\pi / 2, \pi / 2\left[\right.$ for $s_{n}=0$, $\theta_{n-1}-\theta_{n}$ cannot escapes from ] $-\pi / 2, \pi / 2\left[\right.$ for any $s_{n} \in\left[0, L_{n}\right]$. The oriented normal to $\mathcal{C}_{n-1}, v_{n-1}$, is given by

$$
\sqrt{1+d_{n}^{2} \kappa_{n}^{2}} v_{n-1}=-d_{n} \kappa_{n} \tau_{n}+v_{n}
$$

and the signed curvature $\kappa_{n-1}$ of $\mathfrak{C}_{n-1}$ is, after some calculations,

$$
\kappa_{n-1}=\frac{1}{\sqrt{1+d_{n}^{2} \kappa_{n}^{2}}}\left(\kappa_{n}+\frac{d_{n}}{1+d_{n}^{2} \kappa_{n}^{2}} \frac{d \kappa_{n}}{d s_{n}}\right) .
$$

Since $\theta_{n-1}-\theta_{n}$ remains in ] $-\pi / 2, \pi / 2$, the unitary tangent vector $\tau_{n-1}$ has the good direction, i.e., $P_{n-2}=P_{n-1}+d_{n-1} \tau_{n-1}$. The analysis can be continued for $P_{n-2}, \ldots, P_{0}$ and $Q$. This proves (i), (ii) and (iii). 
Assertion (iv) comes from the following formula derived from (32) and (31) $(i=1, \ldots, n)$ :

$$
\kappa_{i-1}=\frac{1}{\sqrt{1+d_{i}^{2} \kappa_{i}^{2}}}\left(\kappa_{i}+\frac{d_{i}}{1+d_{i}^{2} \kappa_{i}^{2}} \frac{d \kappa_{i}}{d s_{i}}\right)
$$

where $s_{i-1}$ is the natural parameterization of $\mathfrak{C}_{i-1}$ defined by

$$
d s_{i-1}=\sqrt{1+d_{i}^{2} \kappa_{i}^{2}} d s_{i}
$$

Consequently, $\kappa_{i}$ is an algebraic function of $\kappa_{n}$ and its first $n-i$ derivatives with respect to $s_{n}$. Moreover, the dependence with respect to $\frac{d^{n-i} \kappa_{n}}{d s_{n}^{n-i}}$ is linear via the term

$$
\frac{d_{i+1}}{\left(1+d_{i+1}^{2} \kappa_{i+1}^{2}\right)^{3 / 2}} \frac{d_{i+2}}{\left(1+d_{i+2}^{2} \kappa_{i+2}^{2}\right)^{3 / 2}} \cdots \frac{d_{n}}{\left(1+d_{n}^{2} \kappa_{n}^{2}\right)^{3 / 2}} \frac{d^{n-i} \kappa_{n}}{d s_{n}^{n-i}}
$$

The map of assertion (iv) has a triangular structure with a diagonal dependence that is linear and always invertible: it is a global diffeomorphism.

Proof of proposition 5 Denote by $\left(\tilde{x}_{n}, \tilde{y}_{n}\right)$ and $\left(\bar{x}_{n}, \bar{y}_{n}\right)$ the cartesian coordinates of $\tilde{P}_{n}$ and $\bar{P}_{n}$, the initial and final positions of $P_{n}$. There always exists a smooth planar curve $\mathcal{C}_{n}$ with a natural parameterization $s_{n} \rightarrow P_{n}\left(s_{n}\right)$ satisfying the following constraints:

- $P_{n}(0)=\tilde{P}_{n}$ and $P_{n}\left(L_{n}\right)=\bar{P}_{n}$ for some $L_{n}>0$.

- the direction of tangent at $\tilde{P}_{n}$ (resp. $\left.\bar{P}_{n}\right)$ is given by the angle $\tilde{\theta}_{n}\left(\right.$ resp. $\left.\bar{\theta}_{n}\right)$;

- the first $n$ derivatives of the signed curvature $\kappa_{n}$ at points $\tilde{P}_{n}$ and $\bar{P}_{n}$ have prescribed values.

According to (iii) and (iv) of the above lemma, the initial and final values of the angles $(i=1, \ldots, n)$ $\theta_{i-1}-\theta_{i}$ and $\phi$ define entirely the initial and final first $n$ derivatives of $\kappa_{n}$. It suffices now to choose a smooth function $[0, T] \ni t \rightarrow s_{n}(t) \in\left[0, L_{n}\right]$ such that $s_{n}(0)=0, s_{n}(T)=L_{n}$ and $\dot{s}_{n}(0)=\dot{s}_{n}\left(L_{n}\right)=$ 0 , to obtain the desired control trajectory via the relations (the notations are those of the above lemma):

$$
\left\{\begin{aligned}
\dot{s}_{0}=u_{1} & =\left(\prod_{i=1}^{n} \sqrt{1+d_{i}^{2} \kappa_{i}^{2}}\right) \dot{s}_{n} \\
u_{2} & =\left(\prod_{i=1}^{n} \sqrt{1+d_{i}^{2} \kappa_{i}^{2}}\right) \frac{d_{0}}{1+d_{0}^{2} \kappa_{0}^{2}} \frac{d \kappa_{0}}{d s_{0}} \quad \dot{s}_{n} .
\end{aligned}\right.
$$

